\title{
Anatomical Properties and Physiological Correlates of the Intrinsic Connections in Cat Area 18
}

\author{
J. A. Matsubara, ${ }^{1,3}$ M. S. Cynader, ${ }^{2,3}$ and N. V. Swindale ${ }^{3}$ \\ Departments of 'Anatomy, ${ }^{2}$ Physiology, and ${ }^{3}$ Psychology, Dalhousie University, Halifax, B3H 4H7, Nova Scotia, Canada
}

\begin{abstract}
After making a map of response properties of neurons in a roughly $3 \times 4 \mathrm{~mm}$ region of area 18 in the cat, we injected wheat-germ agglutinin horseradish peroxidase (WGA-HRP) and succinylated concanavalin $A$ (Con $A$ ) into physiologically identified regions of the map. We observed up to 10 patches of retrogradely labeled cells surrounding each injection site. The majority of the patches occurred within $1.4 \mathrm{~mm}$ of the center of the injection site, but rare patches were found as far as $3.4 \mathrm{~mm}$ from the injection site. The mean center-to-
\end{abstract} center spacing of the intrinsic patches was about $1 \mathrm{~mm}$, while the mean distance between the center of the injection site and the nearest patches was less than $1 \mathrm{~mm}$. The labeled cells included both nonpyramidal and pyramidal types and were found in all layers, although they were usually most dense in layers II-IV. Between $2 \%$ and $9 \%$ of the cells within a cortical column were labeled after a single injection of WGA-HRP or Con A into area 18. Injections of different tracers into 2 neighboring areas resulted in a uniform and less patchy distribution of labeled cells, which suggests that the patches observed after a single injection were only a portion of a continuous horizontal system of interconnections. The patterns and positions of the intrinsic patches were compared to the distribution of the following receptive-field properties: preferred orientation, receptive-field location, and eye preference. The preferred orientations of the recording sites within the injected and labeled areas were different and, most frequently, orthogonal to each other. This is a highly specific projection, since regions with orientation values like those of the injection site were "within range," yet not labeled. We were unable to detect any relationship between the ocular preferences of the injected and labeled cell regions. Injections into areas predominantly driven by the contralateral eye resulted in labeled regions exhibiting varied eye preference distributions. In some animals they were like the injection site and in others there were equal numbers of contra- and ipsilateral eye-dominated regions.

The overall distribution of the patches around the injection site was elongated along the anterior-posterior cortical axis of the brain. The patches extended further in the posterior than the anterior direction. These observations appear to be related to the finding that the cortical magnification factor

\footnotetext{
Received June 20, 1986; revised Aug. 26, 1986; accepted Oct. 2, 1986.

We thank Julia Hickey, Lori Colpitts, and Trudy Tilley for their expert technical assistance. This work was supported by the Medical Research Council of Canada.

Correspondence should be addressed to Dr. Joanne Matsubara, Department of Anatomy, Faculty of Medicine, Dalhousie University, IIalifax, Nova Scotia, B3H 4H7, Canada.

Copyright (C) 1987 Society for Neuroscience $0270-6474 / 87 / 051428-19 \$ 02.00 / 0$
}

is greater along the anterior-posterior than the medial-lateral axis of area 18. The elongation in the distribution of the patches effectively compensates for the functional asymmetry in the magnification factor, so that a population of connected cells represents a roughly circular region of visual space.

It is now well established that cortical structures have a modular organization in which cells occupying the same vertical column of tissue have similar physiological response properties (Mountcastle, 1957). Anatomical studies indicate that a modular organization exists also in the structure of cortcx. Studics on the connectivity of both sensory and nonsensory cortical areas have revealed that thalamocortical and intercortical connections are distributed in bands, stripes, or patches (Hubel and Wiesel, 1972; LeVay et al., 1975; Goldman and Nauta, 1977; Imig and Brugge, 1978; Goldman-Rakic and Schwartz, 1982; Bullier et al., 1984; Symonds and Rosenquist, 1984).

Recently, another striking example of morphological organization that is both modular and periodic has been observed in the pattern of intrinsic, local connections within visual cortex. With the use of tract-tracing methods, such as the transport of horseradish peroxidase (HRP) or radioactive amino acids, it has been discovered that the anatomical connections within a cortical area are not uniformly distributed, but rather grouped into "patches" or "clusters" (Rockland et al., 1982; Sesma et al., 1984; Matsubara et al., 1985; Matsubara and Phillips, 1986). The patches are components of an anatomical network that spreads horizontally across the cortex and joins together isolated clusters of cells in different columns. While it is likely that this network forms a structural basis for the internal processing of information among columns in the cortex, the exact significance of the connections is not yet apparent.

Although many recent studies have focused on either the functional or the anatomical properties within visual cortex, few have attempted to determine both within the same region. A fundamental question that remains unanswered is whether the "patchy" networks of interconnections, as revealed by retrograde tracers, correlate with particular aspects of cortical functional organization revealed by electrophysiological recordings. For instance, are the patchy connections made between cells with similar or different functional properties?

The answer to this question demands knowledge of which regions are locally connected, as well as of the functional properties within the regions, and so a combination of methods has generally been employed. In the tree shrew, Rockland et al. (1982) combined the methods of HRP histochemistry and ${ }^{14} \mathrm{C}$-2-deoxyglucose autoradiography to determine whether the 
patterns of labeling obtained from a local HRP injection coincided with the patterns of metabolic activity resulting from visual stimulation with stripes of a single orientation. The authors found no consistent relationship between the 2 patterns: There were regions of overlap as well as other areas that contained either one or the other tracer. However, the conclusions were ambiguous since damage to the tissue at the site of the injection obscured the autoradiographic label and hence the functional characteristics of the cells within the injection site were not known.

In another series of experiments, Rockland and Lund (1983), Livingstone and Hubel (1984), and Rockland (1985) compared the intrinsic levels of a metabolic enzyme, cytochrome oxidase, with the labeling pattern produced by injections of HRP in both V1 and V2 of the primate. It has previously been shown that high levels of cytochrome oxidase activity in visual cortex are specific markers for regions with nonoriented, monocular, and color-specific physiological responses (Horton and Hubel, 1981; Livingstone and Hubel, 1982). Livingstone and Hubel (1984) showed that areas of dense cytochrome staining were reciprocally connected, as were areas with weak cytochrome oxidase staining. However, areas exhibiting weak cytochrome oxidase staining were not connected to areas with strong staining. Rockland and Lund (1983) also correlated the pattern of intrinsic connections with that of cytochrome oxidase staining, but did not find the same degree of specificity, perhaps because of the larger size of their HRP injections.

A purely physiological approach, using simultaneous recordings, was taken to study correlations in the visually driven impulse activity of pairs of neurons located in the same cortical layer but separated laterally by varying distances (Ts'o et al., 1986). Results from this study showed positive correlations in firing between cells with similar preferred orientation values, suggesting either a direct excitatory connection or common inputs (e.g., from the lateral geniculate nucleus or other cortical areas). These findings suggested preferential interconnections between neurons with similar orientations. However, theoretical studies have indicated that the cross-correlation method greatly emphasizes excitatory interactions over inhibitory ones (Aertsen and Gerstein, 1985). In addition, further experiments by Ts'o et al. (1985) showed that the correlated pairs of cells were not monosynaptically coupled to each other, since direct excitation of 1 cell by application of glutamate did not cause correlated firing of the cell at the other recording site. This finding suggests that any positive correlation is not due to a direct excitatory connection between cells with similar preferred orientation, but rather to common input.

We have adopted a more direct approach to the identification of the functional significance of the patchy, intrinsic connections. We first studied the response properties of neurons and their distribution across approximately a $3 \times 4 \mathrm{~mm}$ area of cortical surface (Cynader et al., 1987). Next, we injected either wheat-germ agglutinin conjugated to horseradish peroxidase (WGA-HRP) or succinylated concanavalin A (Con A) into physiologically identified loci. Response properties of cells within the injection site were compared with those of the areas containing retrogradely labeled neurons. Our results indicate that, in area 18 , the intrinsic networks connect neurons with different preferred orientations. Our results also indicate a relationship between the extent and distribution of the labeled patches and the anisotropic visual-field topography of area 18 . The intrinsic connections, however, appear to be independent of other receptive-field properties, such as ocular dominance, preferred direction of motion, and receptive-field size. Measurements of the number, periodicity, and distribution of the intrinsic patches surrounding an injection site are presented.

A preliminary report of these results has appeared (Matsubara et al., 1985).

\section{Materials and Methods}

Physiological recordings. Area 18 in the cat provides a cortical surface that is flat and easily accessible for mapping studies. We recorded unit clusters from 38 adult cats. The detailed results of the physiological experiments are described in the preceding papers (Cynader et al., 1987; Swindale et al., 1987). Animals were initially anesthetized with intravenous pentobarbitol sodium. Atropine and dexamethasone were administered to reduce salivation and brain edema, respectively. A tracheotomy was performed under surgical anesthesia and the animal was then placed in a stereotaxic frame. We next paralyzed the animal with intravenous gallamine triethiodide, and artificial respiration was begun. The end-tidal $\mathrm{pCO}_{2}$ was monitored continuously and maintained near $4.0 \%$ by adjustments of the rate and volume of the respiratory pump. Intravenous pentobarbitol was discontinued and the animal was kept lightly anesthetized with a mixture of nitrous oxide and oxygen (70:30). EEG and EKG were monitored routinely. Areae centrales were plotted on a tangent screen using a reversing ophthalmoscope. Contact lenses with $4 \mathrm{~mm}$ artificial pupils were chosen by retinoscopy to focus the eyes on a tangent screen $145 \mathrm{~cm}$ distant.

A craniotomy and duratomy were performed over the region of area 18 that represents the lower visual fields. A Davies chamber was attached to the skull over the opening, using methylacrylate and skull screws. The Davies chamber was filled with silicone oil and substantially eliminated pulsations of the brain due to respiratory movements and heartbeat. The surface of the brain and a $1 \mathrm{~mm}$ scale bar placed adjacent to it were photographed with $35 \mathrm{~mm}$ positive film. The slide was then projected onto acetate film and used to identify and mark multiunit recording locations, tracer injection sites, and landmark injection sites.

Multiunit recordings were made at each penetration at a dcpth approximately $300-600 \mu \mathrm{m}$ from the pial surface. Penetrations were spaced about $200-300 \mu \mathrm{m}$ apart in an array roughly $3 \times 4 \mathrm{~mm}$ in area. The electrode was positioned on the cortical surface using visual inspection through a dissecting microscope, and the position of each recording locus was recorded relative to the blood vessel pattern on a photograph of the brain surface. At each site we recorded the following properties: ocular dominance, preferred orientation, preferred direction of motion, receptive-field-center location, and receptive-field size.

Microinjections of tracers were made during or upon completion of the recording sessions. We report here the results of injections of WGAHRP (either 1\% WGA-HRP in saline: cases 6SP, 11SP, 12SP, 13SP, $19 \mathrm{SP}, 21 \mathrm{SP}, 22 \mathrm{SP}, \mathrm{CO} 1,34 \mathrm{SP}, 35 \mathrm{SP}, 41 \mathrm{SP}$; or 0.5\% WGA-HRP in saline: 42SP, 43SP); HRP (20\% in saline: 4SP), or succinylated Con A (1\% in salinc: IM4, 42SP, 43SP) into identificd locations within arca 18. Injections were made by pressure through glass micropipettes (inner tip diameter, 8-10 $\mu \mathrm{m}$ ). Injections of fluorescent markers (rhodaminelabeled latex microspheres and/or bisbenzimide; $1 \%$ in sterile saline) were made and documented on the photograph to allow alignment between the charts of sections and the location of the recording sites also documented on the photograph of the brain surface.

The volume of tracer injected was between 10 and $20 \mathrm{nl}$, as measured by observing the meniscus level of the micropipette before and after the injection. Following a survival time (under anesthesia) of 24-36 hr, the animals were deeply anesthetized and perfused with phosphate buffer $(0.1 \mathrm{M}, \mathrm{pH} 7.2$, with $0.5 \%$ sodium nitrite), followed first by either $4 \%$ buffered paraformaldehyde or a mixture of $2.5 \%$ buffered paraformaldehyde and $0.5 \%$ glutaradehyde, and then by $10 \%$ buffered sucrose. Blocks of tissue were cut in the horizontal plane, tangential to the surface of area 18 , so that the pattern of labeling surrounding the injection site could be directly compared with the sites of the physiological recordings, as documented on photographs of the exposed brain surface.

In other cases (21SP, 22SP, CO1), the blocks were sectioned in the coronal plane to enable us to study the laminar and columnar organization of the intrinsic patches of label. The tissue was sectioned on a freezing microtome at a thickness ranging between 50 and $80 \mu \mathrm{m}$.

Sections were processed in 1 of 3 ways: (1) A 1 -in-3 series was reacted, using the tetramethylbenzidine (TMB) method, for HRP (Mesulam, 1978). Another one-third was processed for Con A immunohistochem- 

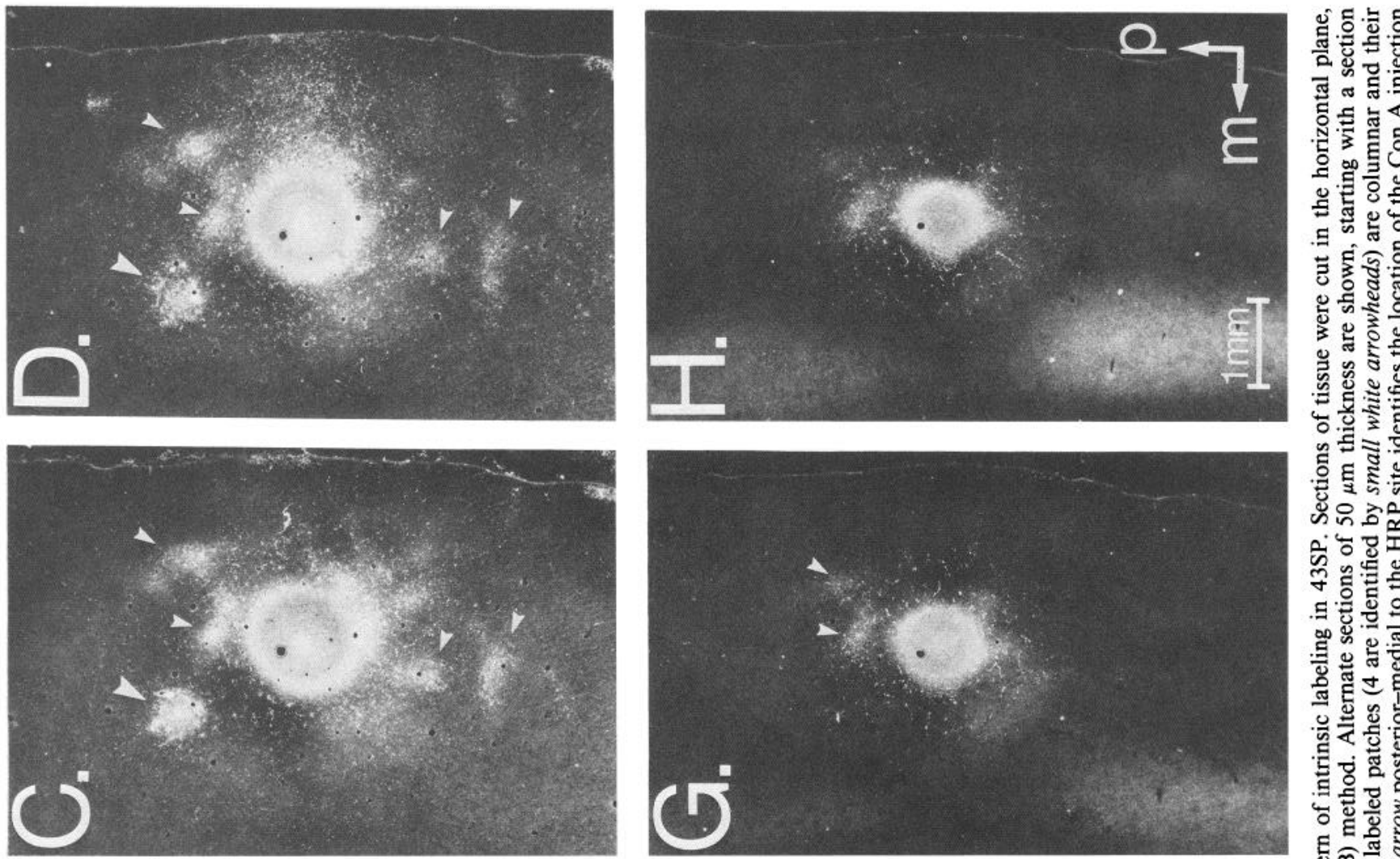

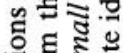

过的的

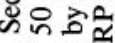

능

吗

플

on

5 㖓

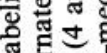

a

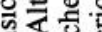

웜

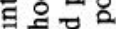

पै

트의

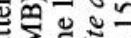
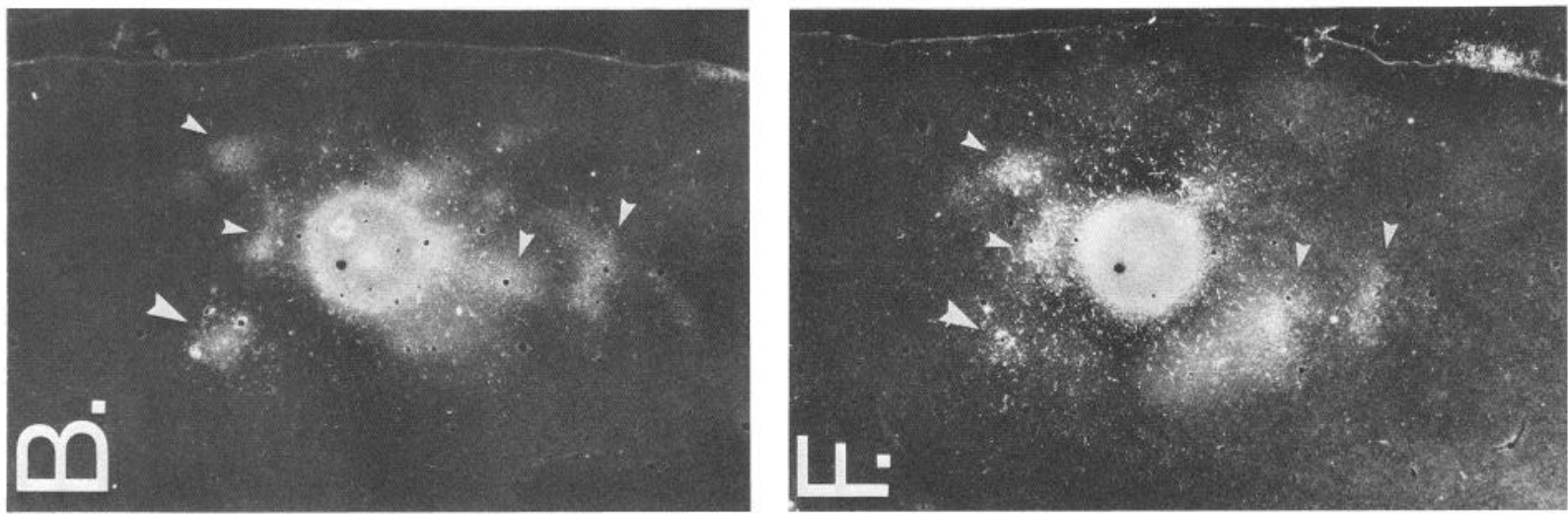

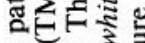

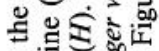
둔 5. o

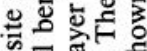

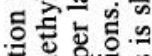

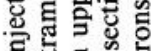

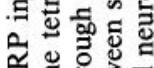

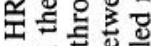
5. \% 3.0

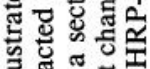
总导步

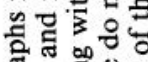
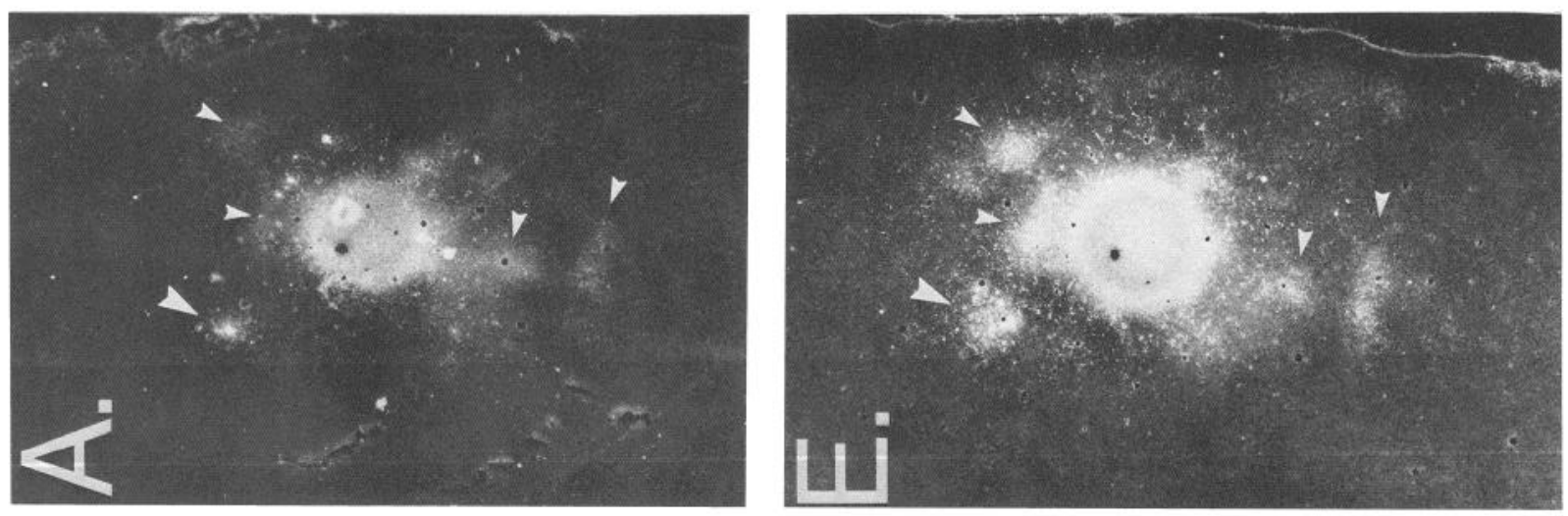
的央 운

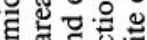
는 을 a. 물 音导守 ด 9 㟧 - 可은 들 맬. 농영. 
istry (Nance, 1984). The last third was processed for both tracers. Sections were first reacted with the TMB method and stabilized using a modification of the method of Rye et al. (1984) in which sections were incubated in a solution consisting of $0.05 \%$ diaminobenzidine (DAB), $0.02 \%$ cobalt chloride, $0.02 \%$ nickel ammonium sulfate, and $0.001 \%$ $\mathrm{H}_{2} \mathrm{O}_{2}$ in phosphate buffer for $10-20 \mathrm{~min}$ at room temperature. This second incubation produced an insoluble reaction product, which, we found, had a greater sensitivity than that achieved in the original method of Rye et al. (1984) or when using the DAB reaction alone (D. M. Nance and J. A. Matsubara, unpublished observations). After completion of the stabilization method, the sections were incubated in antisera solutions to allow visualization of the neurons retrogradely labeled with Con A.

Succinylated Con A has been shown to be a sensitive retrograde and anterograde tracer in the mammalian central nervous system (Nance, 1984). Sections of tissue were rinsed 3 times in PBS (pH 7.3), then incubated in a primary antibody made in rabbit against Con A (Sigma) for $18-24 \mathrm{hr}$ at room temperature. The optimal dilution of the antiserum was 1:40,000 (Sigma). The primary antiserum was tagged sequentially with biotinylated goat anti-rabbit (secondary) antibody and avidin DH: biotinylated HRP solution (VectaStain). Next, the peroxidase label was visualized using a carbazole (Pearse, 1972) or a DAB chromogen.

Charts of the labeled neurons, injection sites, and marker sites were drawn at both low and high power using a drawing tube connected to either a Wild dissecting microscope, a Leitz Dialux, or a Nikon Optiphot compound microscope. In the charts shown in Figures 8, 12-15, the labeled cells were identified under bright-field illumination and their positions were recorded using a pair of stage potentiometers connected to a $X-Y$ plotter. Charts of adjacent sections were aligned with each other using at least 3 injection sites (usually 2 tracer injection sites and 1 landmark injection site). The outline of each tissue section, as well as the cross sections of major blood vessels, were additional cues that were used to align given pairs of consecutive sections. Because we recorded in the upper layers of area 18 (primarily layers II and III), we compared the labeling pattern from these layers with the physiological responses. Coronal sections of area 18 (Fig. 3) indicated that the boundary between layers III and IV was typically midway between the surface and white matter (Harvey, 1980; Humphrey et al., 1985). The drawings of laminar boundaries in Figure 3 were based on comparisons between sections stained for cytochrome oxidase and for Nissl substance (Price, 1984).

A cumulative chart of sections corresponding to layers I-III and upper IV was produced on clear acetate. Since accurate alignment of the physiological response properties and the anatomical charts of labeled cell bodies was critical, we used a variety of criteria to ensure accurate alignment. The chart was aligned with the photograph of the brain surface using the marker and tracer injection sites, as well as the outline of the tissue sections, as guides. In some cases we had additional alignment cues, such as the subtle indentations in the tissue caused by large blood vessels running across the cortical surface. We were able to test the accuracy of the alignment between the cumulative chart and the photo of the brain surface (which included the position of the recording loci relative to the injection sites) in a few cases in which small hemorrhages were caused by the tip of the electrode hitting branches of blood vessels deep in the tissue during the physiological recording sessions. Since the electrode was positioned on the cortical surface and advanced into the cortex under visual control, we immediately knew of any damage to underlying blood vessels, and siles of bleeding were recorded on the photo of the brain surface. Some (but not all) of these sites were visible as small scars (i.e., spots in which the blood did not clear during perfusion) after the tissue was processed for HRP histochemistry. After alignment of the chart with the photograph these scars fells within $\pm 50 \mu \mathrm{m}$ of their recorded positions.

Outlines of labeled cell regions consisting of a minimum of 5 cells/ $0.04 \mathrm{~mm}^{2}$ were drawn and the numbers of the recording loci falling within the laheled areas were tabulated (Figs. 10, 16, 17). Next, we compared the ocular dominance, preferred orientation, and direction of motion of the units recorded within the injection site and labeled areas.

Preferred orientation of cells within the injection site and the labeled cell areas were plotted in circular histograms. Because the distribution of preferred orientation angles was circular, the double-angle method of Krumbein (Moore, 1980; Batschelet, 1981) was used to calculate the means and standard deviations of the samples. A detailed description of the method used to calculate the mean orientation angles is given in an accompanying paper (Swindale et al., 1987; see Swindale, 1982, 1985).

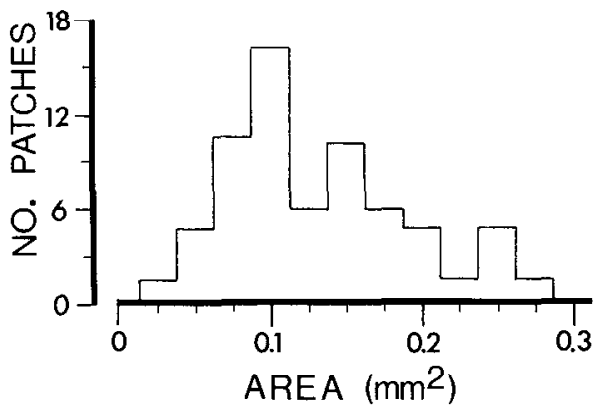

Figure 2. Histogram of the cortical surface area $\left(\mathrm{mm}^{2}\right)$ occupied by individual patches arising from 5 injection sites (IM4C, IM4H, 43SPH, 43SPC, and 42SPH). Diameters of the injection sites were approximately $800 \mu \mathrm{m}$. The peak in the histogram occurs at $0.1 \mathrm{~mm}^{2}$.

Measurements of cortical area occupied by the labeled patches, as well as the overall area within which labeling occurred, were made using Videoplan software. We found no difference between either the size of the individual patches or the overall area of label using Con $\mathrm{A}$ or WGA-HRP as tracers.

\section{Results}

General description of the pattern of intrinsic labeling Shapes and sizes of intrinsic patches

The patterns of labeling produced by WGA-HRP and succinylated Con A were similar and consisted of retrogradely filled cell bodies surrounded by a granular reaction product characteristic of anterograde and/or collateral transport. The labeled cells were grouped together into patches or clusters surrounding the injection site. The patches were particularly evident in sections of tissue cut tangential to the cortical surface (Fig. 1). Each patch could be identified and followed through serial tangential sections. The number of patches surrounding an injection site varied between 2 and 10 (mean, 5.7, SD, 2, $n=15$ ). The mean value was a minimal estimate of the number of intrinsic patches connected to a given cortical locus, presumably because the extent of labeling was dependent on the quality of the perfusion and subsequent histochemical reactions. The distant patches were more susceptible to fading than the ones closer to the injection site, probably because there was less HRP in the cells.

When viewed in sections cut in the tangential plane (Fig. 1), the patches were quasicircular in outline, with a diameter of roughly $350 \mu \mathrm{m}$, and occupied about $0.1 \mathrm{~mm}^{2}$ of cortical area (Fig. 2). Taken together, the patches occupied between 15 and $28 \%$ (mean, $19.5 \% \pm 4 \%, n=11$ ) of the surrounding "available" area. The "available" cortical area was defined by first delimiting the entire region containing labeling. The area of the injection site was then subtracted from this value to give a measure of the "available" cortical area within which we could expect to finding labeling.

\section{Laminar distribution}

Sections of tissue cut in the coronal plane revealed labeled cells in vertical columns running through all 6 cortical layers (Fig. 3 ). While the greatest number of cells was usually found in layers II-III, their density was highest in layer IV, then II-III, V, VI, and I. Both nonpyramidal and pyramidal cell types were labeled (Fig. 4).

The number of cells labeled within the patches surrounding an injection site varies between $2 \%$ and $9 \%$ (mean, $4.8 \% \pm 2 \%$, $n=14$ ) of the total number of cells in an equivalent volume of 

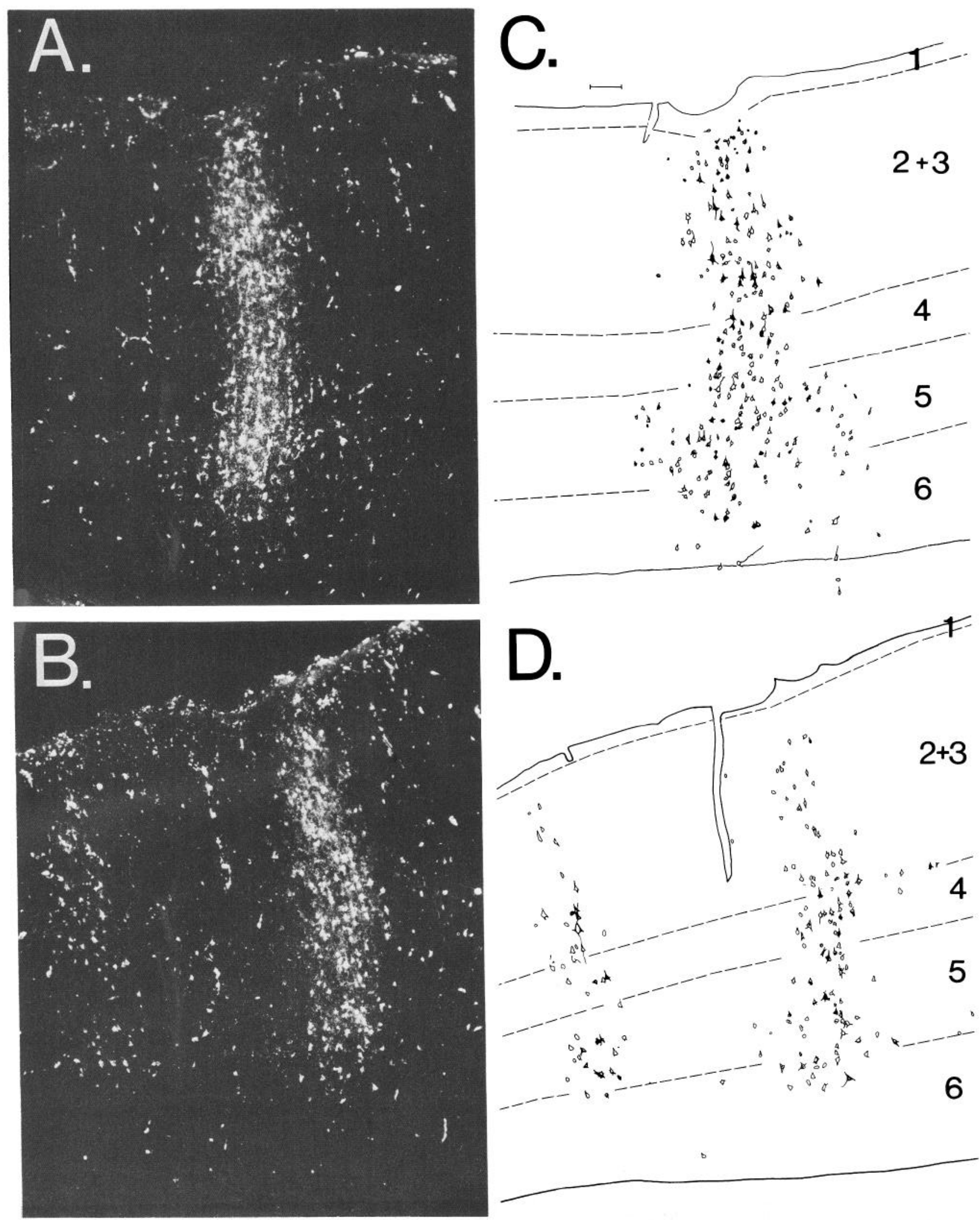

Figure 3. Dark-field photomicrographs $(A, B)$ of 2 adjacent coronal sections through the same labeled patch. The edge of another patch can be seen on the left in $B$. The camera lucida drawings $(C, D)$ reveal that the labeled neurons are in all cortical laminae. The density of labeled neurons varies between laminae. Layer IV has the highest density of labeled neurons. Note that pyramidal as well as nonpyramidal cell types are labeled. Scale bar in $C, 100 \mu \mathrm{m}$. 

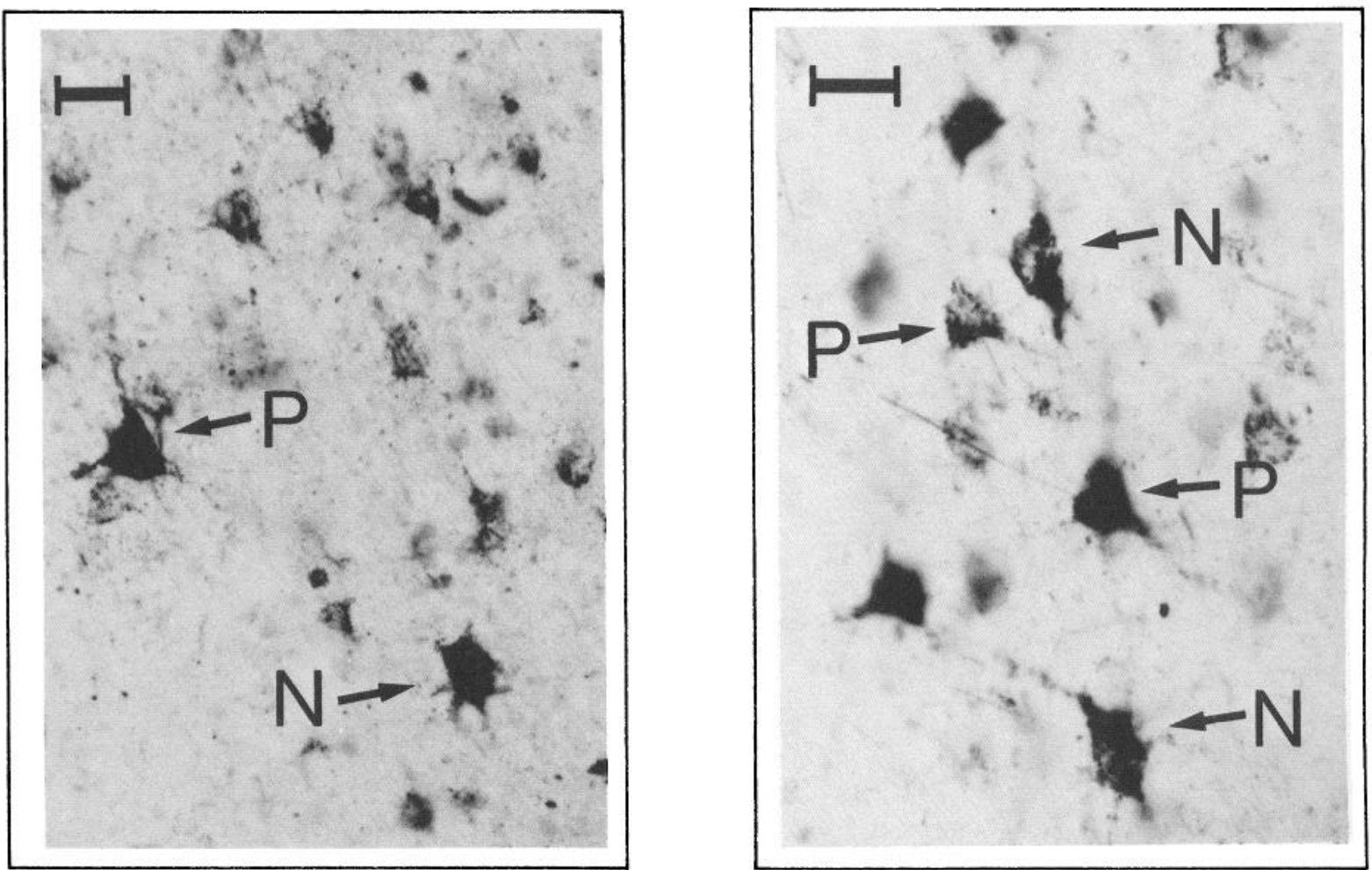

Figure 4. Bright-field photomicrographs of WGA-HRP filled neurons in 2 intrinsic patches labeled after injections into area 18. The sections illustrated here were processed using the TMB method and were not counterstained. Some of the cells are pyramidal $(P)$, while others are fusiform or multipolar $(N)$ in shape. The nonpyramidal forms clearly lack a thick apical dendrite characteristic of pyramidal cells. Scale bars, $15 \mu \mathrm{m}$.

cortex $\left(26,000\right.$ cells $\left./ \mathrm{mm}^{3}\right)$ according to the calculations of Beaulieu and Colonnier (1984).

\section{Injection-to-patch and patch-to-patch distances}

The majority of patches occurred within $1.4 \mathrm{~mm}$ of the injection site. However, rare patches of label were found up to $3.4 \mathrm{~mm}$ from the center of the injection site (Fig. $5 A$ ). The patches were periodically distributed with a modal interpatch spacing of $1 \mathrm{~mm}$ (Fig. $5 B$ ). The extent of labeling was greater along the anterior- posterior than the medial-lateral axis of area 18 (Fig. $6 A$ ). Furthermore, along this axis the labeling was usually more extensive in the posterior than in the anterior direction (Fig. 6B).

Physiological responses of cells in the injected, labeled, and control zones

\section{Preferred orientation}

Small injections of tracers were made into areas with similar preferred orientation values. The volume and concentration of
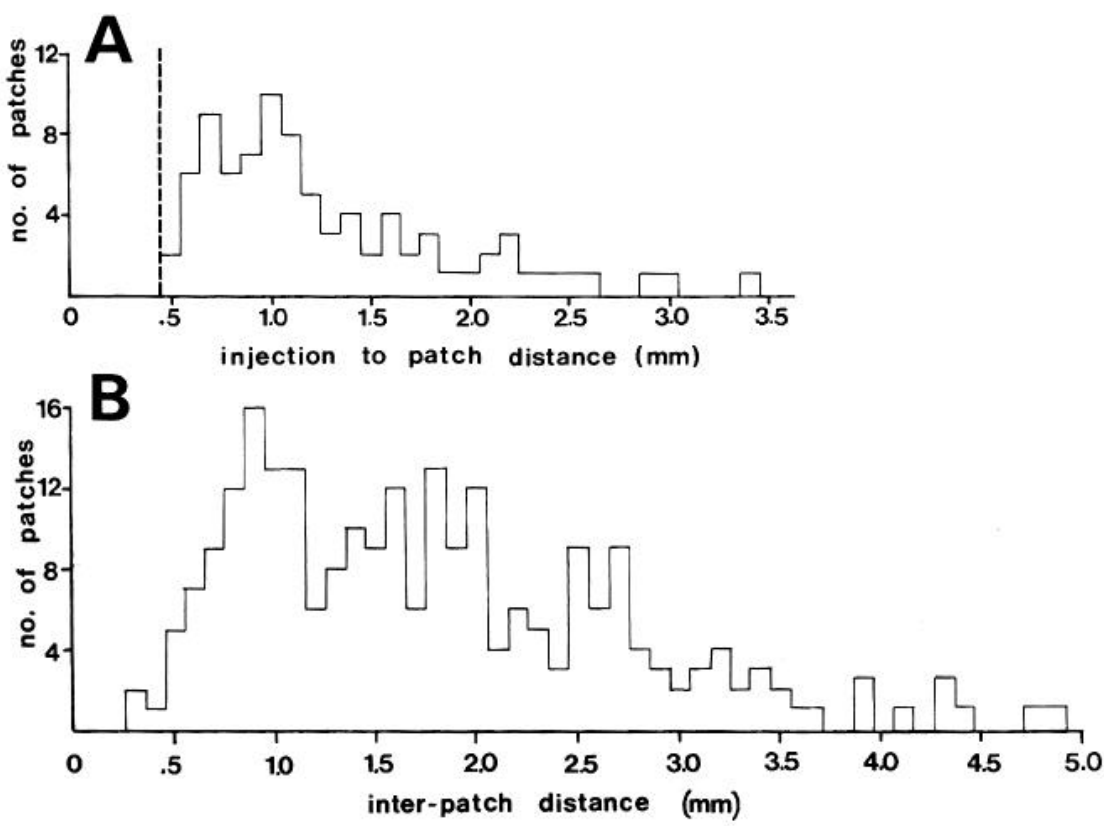

Figure 5. A, Distance between the center of the injection site and each labeled patch from 11 injections. The dashed line at the far left indicates the zone in which we were unable to identify labeled neurons because of the intense reaction product within the core and halo of the injection site itself $(0.45$ $\mathrm{mm}$ or less from the injection center). Although the majority of the intrinsic patches occur within $1.4 \mathrm{~mm}$ of the injection site, there are rare patches of label visible up to $3.4 \mathrm{~mm}$ from the center of the injection. $B$, Periodicity of patches, illustrating the distance between all pairs of patches surrounding the injection sites in 14 animals. The first peak in the histogram is at $0.9 \mathrm{~mm}$, as determined by spectral analysis. Peaks at multiples of $0.9 \mathrm{~mm}$ are also seen $(1.8$ and $2.7 \mathrm{~mm})$. 


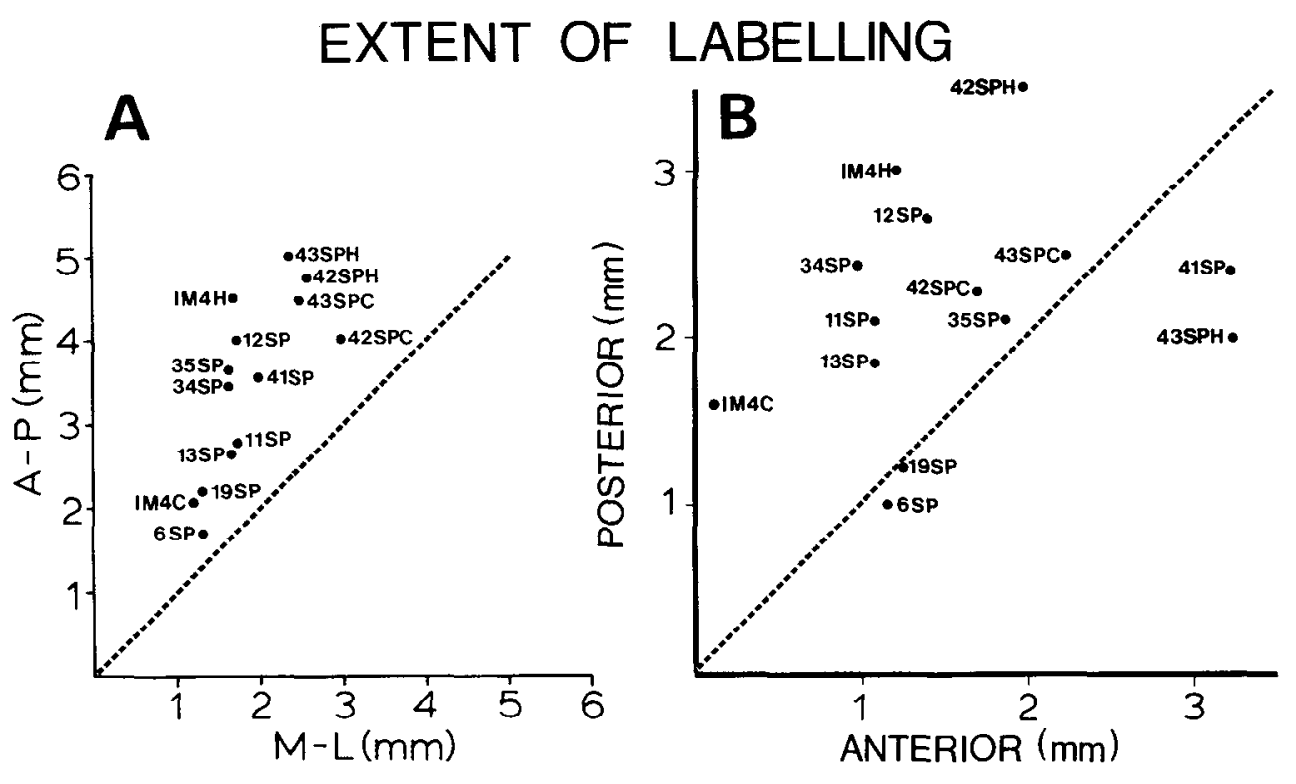

Figure 6. A, Extent of labeling arising from an injection in area 18 is elliptical in overall shape: The distance between the anteriormost and posteriormost patch is always greater than that between the lateralmost and medialmost patches. Note that the maximal extent of labeling in the anterior-posterior direction is $5.0 \mathrm{~mm}$ and only $2.5 \mathrm{~mm}$ in the medial-lateral direction. This represents an anisotropy of $2: 1$ in the former direction. $B$, Along the anteriorposterior axis there was a trend for more of the patches to be situated at greater distances in the posterior than the anterior direction, as is evident from this graph of the maximal spread of labeling in the 2 directions.

the HRP or Con A were adjusted to produce an overall injection site (halo plus core) of less than $1 \mathrm{~mm}$. There were usually between 3 and 5 recording loci within the overall injection site. The mean orientation angle of each injection site was calculated using the double-angle method of Krumbein (Batschelet, 1981; Swindale et al., 1987). Figure 7 illustrates the mean preferred orientation of each injection site. We tried to inject areas with mean preferred orientation values that were different for different animals to see if the distribution of labeling and the preferred orientation of the injected area were correlated.

An example of our results, after the charts of labeled cells were aligned with the maps of preferred orientation values, is shown in Figures 8 and 9. The preferred orientation values obtained in 43SP are represented over each recording site in

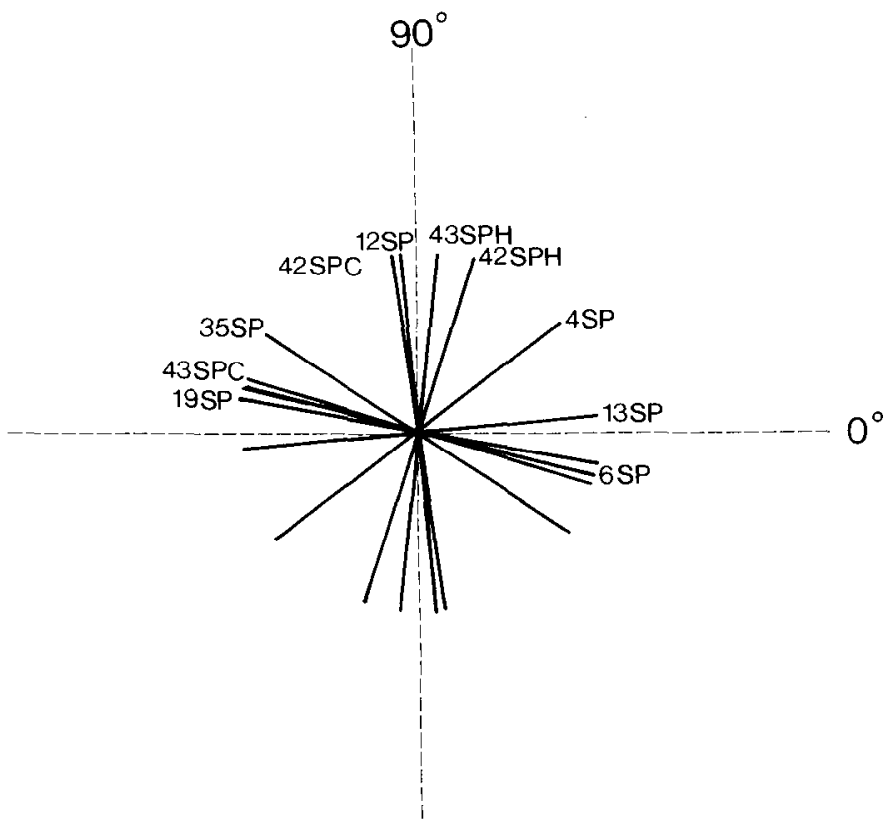

Figure 7. Mean preferred orientation of all physiologically identified injection sites used in this study. Note that the preferred orientations of the injected areas included horizontal, oblique, and vertical values.
Figure $8 \mathrm{~A}$. In this figure the diagonal band devoid of orientation values represents an area from which we were unable to record because of an overlying blood vessel. In Figure $8, B, C$, the outlines surrounding some of the preferred orientation values identify those regions with near-vertical orientations (i.e., an iso-orientation domain spanning orientations between $137^{\circ}$ and $27^{\circ}$, horizontal $=0^{\circ}$ ). The regions outside these outlines represent the orthogonal iso-orientation domain and have preferred orientation values near horizontal (between $138^{\circ}$ and $207^{\circ}$ ). We injected Con $\mathrm{A}$ into a region with preferred orientation values near horizontal (circle $\mathrm{C}$ in Fig. $8 \mathrm{C}$ ). Retrogradely labeled cells were found in patches falling within the iso-orientation domain orthogonal to that of the injection site. Recording sites in regions with 5 or more labeled cells per $0.04 \mathrm{~mm}^{2}$ were scored as labeled and are circled in Figure $8 B$. Labeled areas, which we mapped, were also found outside the region, and in these cases we were unable to evaluate the corresponding physiological response properties.

The orientation values of the injection site, labeled area (projection zone), and surrounding nonlabeled areas (control) are shown in the circular polar graphs in Figure 9 (top). They illustrate that most of the preferred orientations within the labeled areas are different from those of the injection site. This rcsult is also evident in the histograms below the circular graphs. The angular difference between the mean of the injection sample $\left(\bar{\gamma}_{\mathrm{inj}}=161^{\circ}\right)$ and the individual preferred orientations within the injection site $\left(\left|\bar{\gamma}_{\mathrm{inj}}-\gamma\right|\right)$ are shown on the far left. This normalized difference represents the degree of spread in the preferred orientation values within the injection zone. Notice that the overall spread of the injection site does not exceed $\pm 27^{\circ}$. The middle histogram in Figure 9 illustrates the angular difference between the mean orientation value of the injected area and the individual preferred orientations of the labeled regions $\left(\left|\bar{\gamma}_{\mathrm{inj}}-\beta\right|\right)$. This histogram clearly illustrates that the preferred orientations of the labeled areas are not only different from those of the injection site but tend to encompass those orientations representing the larger difference values. The angular difference between the mean preferred orientations of the injected and labeled regions in $43 \mathrm{SPC}$ is $63^{\circ}$.

The specificity of the connections in 43SPC is apparent upon 


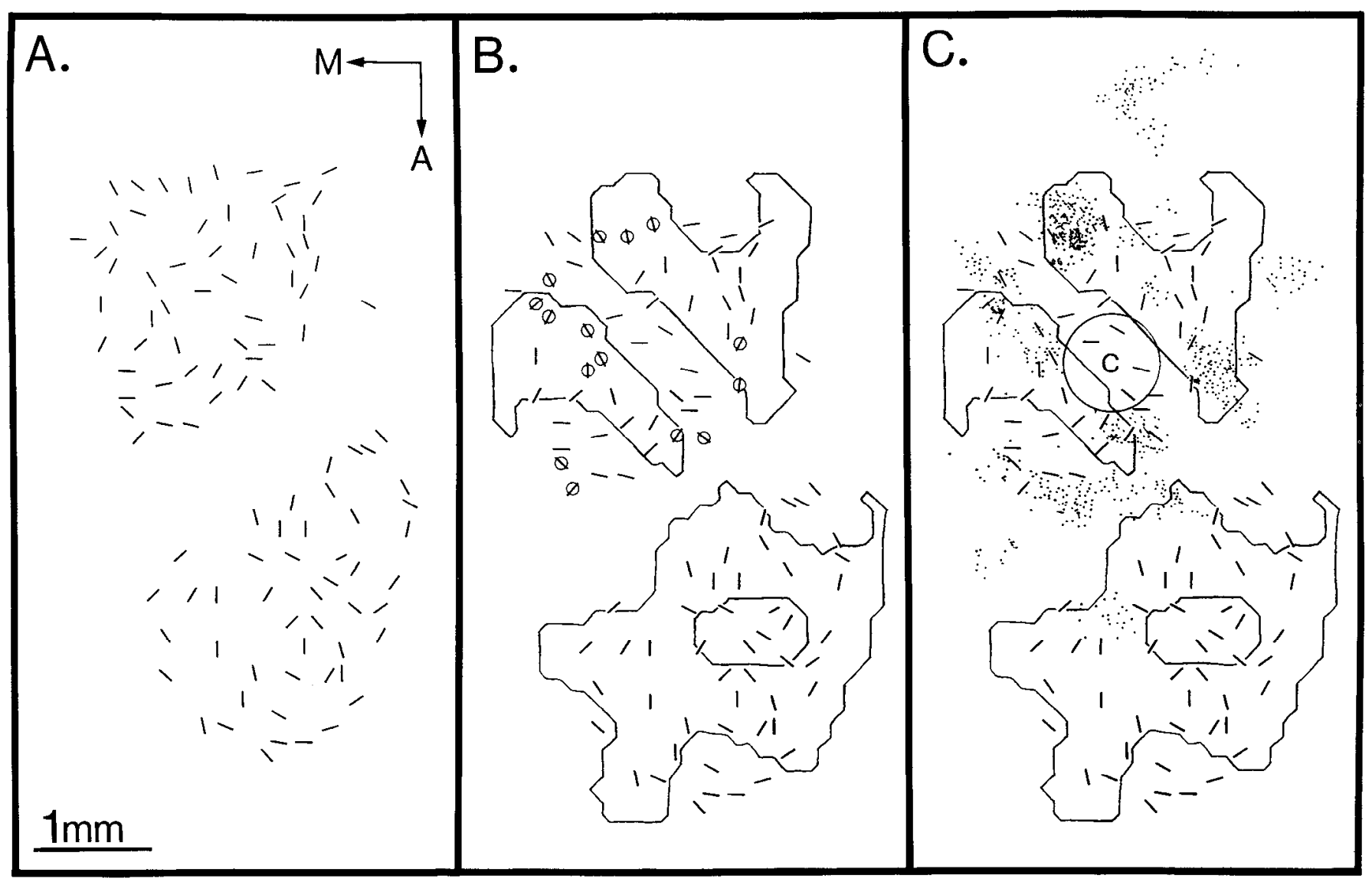

Figure 8. Preferred orientation values of recording sites in 43SP. The lack of recording sites in the center of the map represents a region from which we were unable to record because of a large, overlying blood vessel. $B, C$, Contour lines are drawn around regions with preferred orientation values centered slightly off vertical (range, $37^{\circ}-127^{\circ}$ ). Note that in the posterior portion of the map the vertical and horizontal iso-orientation domains are roughly equal in size and have an overall direction of elongation from posterior-medial to anterior-lateral over the cortical surface. In the anterior portion of the same map there is an unusual asymmetry in the size of the regions possessing horizontal and vertical orientations. An injection of Con A was placed into a horizontally oriented region of the map, as shown in $C$ (circle $C$ ). The circled preferred orientation values in $B$ indicate those areas in which we found more than 5 labeled cells $/ 0.04 \mathrm{~mm}^{2}$. Note that most of the labeling was found in regions within the iso-orientation domain orthogonal to the injected area. In some instances, we found labeling in areas from which we did not record (such as those under blood vessels) and hence we were unable to identify the physiological response properties in these regions.

analysis of the preferred orientations within the control area (i.e., a circular area surrounding and exclusive of the injection site, whose radius is defined as the maximum distance between the center of the injection and the most distant patch of labeling). The circular polar graph on the far right ("Control" in Fig. 9) shows that all preferred orientations, including ones like those in the injection site, were present in the control region. This fact is, again, evident in the histogram below the polar graph, which identifies the normalized difference angles between the mean orientation of the injection site and the individual preferred orientation within the control area $\left(\left|\bar{\gamma}_{\text {inj }}-\alpha\right|\right)$. The control histogram indicates that regions with orientations like those found in the injection site were "within range" of the injection site. In spite of this, however, we found very few regions with "like" oricntation prefercnce labcled.

Figure 10 shows 2 sets of histograms in the same format as was described previously for Figure 9 . In Figure $10 A$, the normalized angular difference values of injected, labeled, and control areas obtained from 7 injections are shown. As in Figure 9 , the histogram on the far left represents the normalized preferred orientations within the injection site. Note that the majority of the preferred orientations within the injected areas fall within $27^{\circ}$ of the mean preferred orientation of these samples. The middle histogram illustrates the normalized angular difference between the injected and labeled areas. Here, as in 43SPC, most of the preferred orientations within the labeled areas were different from those of the injected areas, and tended to encompass those values, producing the larger difference values. The histogram on the far right gives the angular difference between the mean preferred orientation of the injected area and the individual orientations within the control areas. Again, as in 43SPC, regions "within range" of the injection site possessed preferred orientation values that were like those of the injected areas (i.e., with difference values near $0^{\circ}$ ). Yet relatively few areas exhibiting orientations like those of the injection sites were labeled. The majority of the labeled regions were in areas with preferred orientation values that were different from those of the injection sites.

These results contrast with those obtained from injections centered over areas exhibiting a relatively greater range of preferred orientation values. Figure $10 B$ illustrates the normalized angular differences between injected, labeled, and control areas obtained from 5 injections placed in regions with a wide range of orientation values. Unlike Figures 9 and $10 A$, here the nor- 


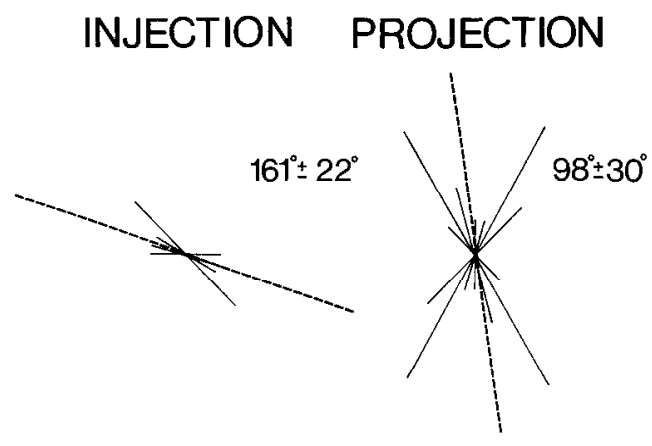

\section{CONTROL}

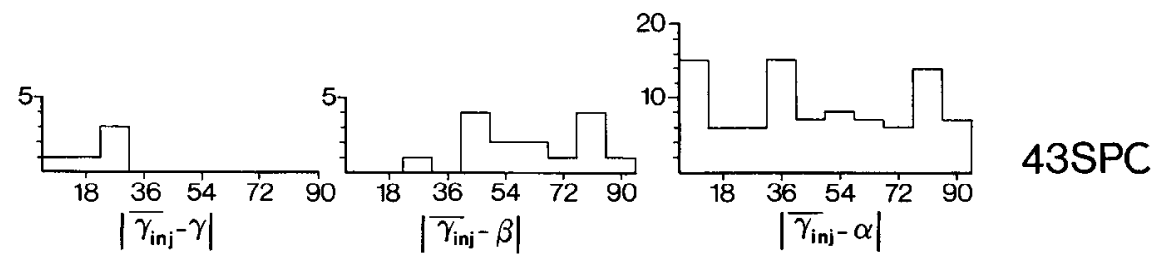

Figure 9. Individual orientation values of the injection site, labeled areas (Projection) and surrounding nonlabeled areas (Control) are shown in polar coordinates. The mean preferred orientation of the injected area was $161^{\circ}\left( \pm 22^{\circ}\right)$ and is indicated by the dashed line. The mean orientation value of the labeled regions was $98^{\circ}\left( \pm 30^{\circ}\right)$. Note that most of the preferred orientations within the labcled regions are different from those in the injection site. The specificity of this connection is evident from the polar graph on the far right, which illustrates the preferred orientations within the surrounding control area. The control area is defined as a circular area (surrounding and exclusive of the injection site) whose radius is the distance between the center of the injection and the most distant patch of labeling. Note all orientations, including ones like those in the injected area, were present and "within range" of the injection site. The normalized angular difference values are shown below the circular polar graphs. These histograms indicate the difference between the mean preferred orientation of the injected area $\left(\bar{\gamma}_{\text {inj }}=161^{\circ}\right)$ and the individual orientations of the injected $\left(\left|\gamma_{\text {inj }}-\gamma\right|\right)$, labeled $\left(\left|\bar{\gamma}_{\text {inj }}-\beta\right|\right)$, and control $\left(\left|\bar{\gamma}_{\text {inj }}-\alpha\right|\right)$ regions. The histogram of the normalized angular difference of the orientations in the injection site represents the degree of spread in the preferred orientation values within the injection zone. Note that the overall spread of the injection does not exceed $\pm 27^{\circ}$. The middle histogram illustrates the normalized angular difference between the injected and labeled regions. This histogram clearly indicates that the preferred orientation of the labeled areas are not only different from those of the injection site, but tend to encompass those orientations representing the larger difference values. The difference between the mean of the injected and the mean of the labeled regions is $63^{\circ}$. The histogram on the far right indicates that all orientations, including ones like those in the injected area (i.e., with difference values near 0 ) were present and "within range" of the injectio site.

malized difference angles between the mean orientation valuc and the individual orientation values within the injection sites were distributed more or less uniformly along the abscissa. Because the injection site included a relatively complete range of orientation values, the la'seled areas, although presumably connected to regions within the injection site with different preferred orientation values, also included a complete range of values. In such cases, the histogram of the angular difference between the mean of the injected sample and the individual preferred orientations of labeled cell areas exhibited more scatter.

\section{Ocular dominance}

We could find no evidence for a correlation between the ocular dominance values of the recording loci within the injected and labeled cell regions. Injections centered over regions that were driven primarily by the contralateral eye (i.e., in areas with ocular dominance values 1-3) resulted in labeled arcas cxhibiting a variety of ocular dominance distributions, as illustrated in Figure 11. If one compares 2 individual cases, 35SP and $42 \mathrm{SPH}$, it is clear that injections centered over areas driven predominantly by the contralateral eye resulted in quite different ocular dominance distributions among the labeled cells in the 2 animals. In 35SP, we found that the labeled areas exhibited responses driven by either eye, whereas in $42 \mathrm{SPH}$, the majority of the labeled areas possessed binocular responses or were driven by the contralateral eye. Attempts to regroup the ocular dominance values into predominantly monocular (groups 1, 2, 6, and 7) and binocular (groups 3-5) categories were equally unsuccessful at revealing correspondences between injected and labeled areas. The majority of the ocular dominance distributions of the labeled areas was not significantly different from the control distributions, regardless of the distribution within the injection site (42SPH, 43SPH, 42SPC, 13SP in Fig. 11).

\section{Receptive-field position}

The representation of the visual field in area 18 was anisotropic, with the magnification factor for elevation in visual space being twice that for azimuth (Tusa et al., 1979; Albus and Beckman, 1980; Cynader et al., 1987). We observed a general elongation in the distribution of labeling along the anterior-posterior axis in all of the cases regardless of the preferred orientation of the injection site (Figs. 6A,7). This was the axis corresponding to elevation in visual space, for which the magnification factor was greatest. Moreover, along the antcrior-posterior axis we observed that the majority of injections resulted in labeling that extended further in the posterior than the anterior direction (Fig. $6 B)$.

The point-spread function, an areal measure of the cortical region within which a given visual receptive-field center is found, was also elongated along the anterior-posterior axis (Cynader et al., 1987). Since the degree of anisotropy in the cortical magnification factor and the asymmetry of the point-spread function varied among individual cases, we examined the degree to which this anisotropy and the distribution of labeling in a given case 
INJECTION
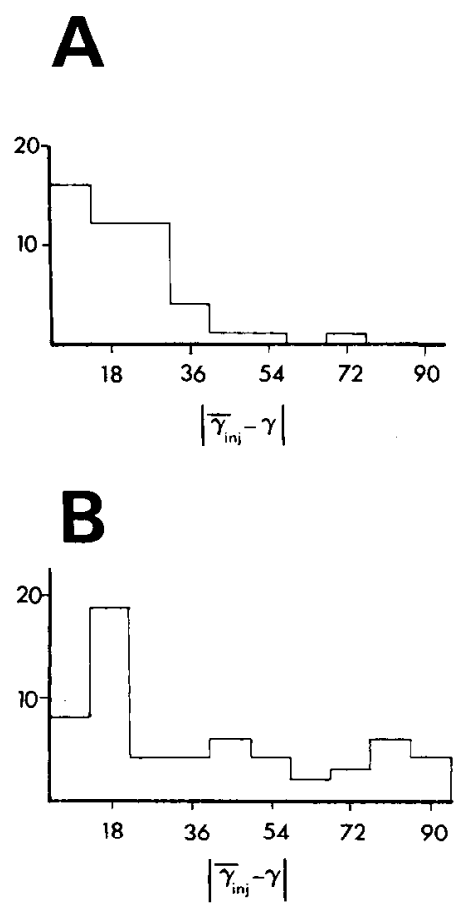

PROJECTION
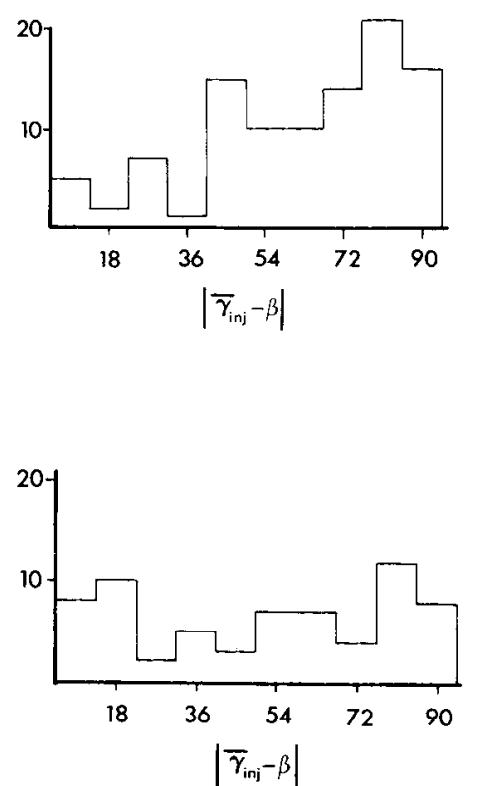

CONTROL
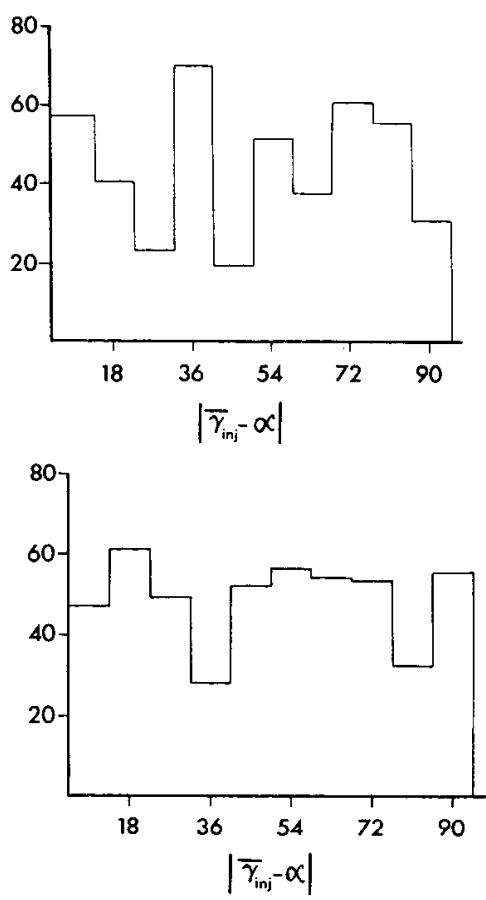

Figure 10. A, Histograms in the same format as those in Figure 9, illustrating results obtained from 7 injections placed into physiologically identified regions of area 18 . The angular difference between the mean preferred orientation of the injection site and the individual orientation values of the injected, labeled, and control areas are shown. The histogram on the far left (Injection) indicates the degrec of sprcad in the preferred orientation values within the injection zone. Notice that, as in Figure 9, the injection sites were confined to cortical areas with a relatively narrow range of preferred orientation values (i.e., $\pm 30^{\circ}$ ). The middle histogram illustrates the angular difference between the mean orientation value of the injected area and the individual orientation values of the labeled regions. Again, as in Figure 9, most of the preferred orientations within the labeled areas were different from those of the injected areas and tended to encompass those values representing the larger difference values. The specificity of the connection is evident from the histogram on the far right, which shows the angular difference between the mean preferred orientation of the injected area and the individual orientations within the control areas. Note that, as in Figure 9, regions "within range" of the injection site possessed preferred orientation values like those of the injected areas (i.e., with difference values near $0^{\circ}$ ). Yet relatively few areas exhibiting orientations like those of the injection site were labeled. $B$, Cumulative histograms obtained from 5 injections placed into regions possessing a wide range of preferred orientation values. As in $A$, the cumulative angular difference between the mean preferred orientation of each injected region and the individual orientation value of the injected, labeled, and control areas are presented. The normalized difference angles of the injected regions are distributed more uniformly than in $A$, indicating that the range of preferred orientation values in the injected zone was broad. The middle histogram illustrates the normalized angular difference between the injected and labeled regions. In contrast to $A$, this histogram indicates that more preferred orientation values representing the smaller difference angles were labeled after injections. Presumably the connections were to areas with different preferred orientations, but because the injected zone includes a more complete range of orientations, the labcled arcas also include a relatively complete range of values.

might be correlated. We found no consistent relationship between the elongation of labeling surrounding an injection site and the individual anisotropy in the magnification factor or elongation of the point-spread function.

\section{Double injections into physiologically identified regions}

Injections centered over regions with overlapping orientation domains

Injections of Con A and WGA-HRP into areas with overlapping prefcrred orientation domains resulted in overlapping zones of labeling (Fig. 12). In one such case, 42SP, the injections were centered over different regions, each consisting of a broad range of preferred orientations centered near vertical, as shown in Figures 13 and 14. The format of these figures is like that of Figure 8; they illustrate the preferred orientation value of each recording site in 42SP. The outlines in Figures 13, B,C and 14, $B, C$, identify those regions with preferred orientation values between $-45^{\circ}$ and $60^{\circ}$ (Fig. 13) and $120^{\circ}$ and $45^{\circ}$ (Fig. 14). The injection of HRP was placed over an area whose mean preferred oricntation was $69^{\circ}\left( \pm 28^{\circ}\right)$, while the Con A was injected into an area with a mean orientation of $93^{\circ}\left( \pm 33^{\circ}\right)$. Both injections labeled areas with a preferred orientation range that peaked near horizontal but with a broad range: Areas with HRP labeling had a mean orientation of $22^{\circ}\left( \pm 36.8^{\circ}\right)$, while the mean orientation of the areas with Con A labeling was $7^{\circ}\left( \pm 35.6^{\circ}\right)$.

In another case (IM4; not shown), the WGA-HRP and Con A injections were separated by $2.2 \mathrm{~mm}$ anteriorly. We observed overlap between 2 pairs of labeled patches. Although we did not record physiological responses in this animal prior to making the injections, we suspect that the 2 injections were placed into regions with overlapping orientation domains, judging from the overlap in the labeling patterns resulting from the 2 tracers.

We observed no difference in the quality or pattern of labeling between cases such as IM4 (in which we did not record) and 


\section{INJECTION PROJECTION CONTROL}
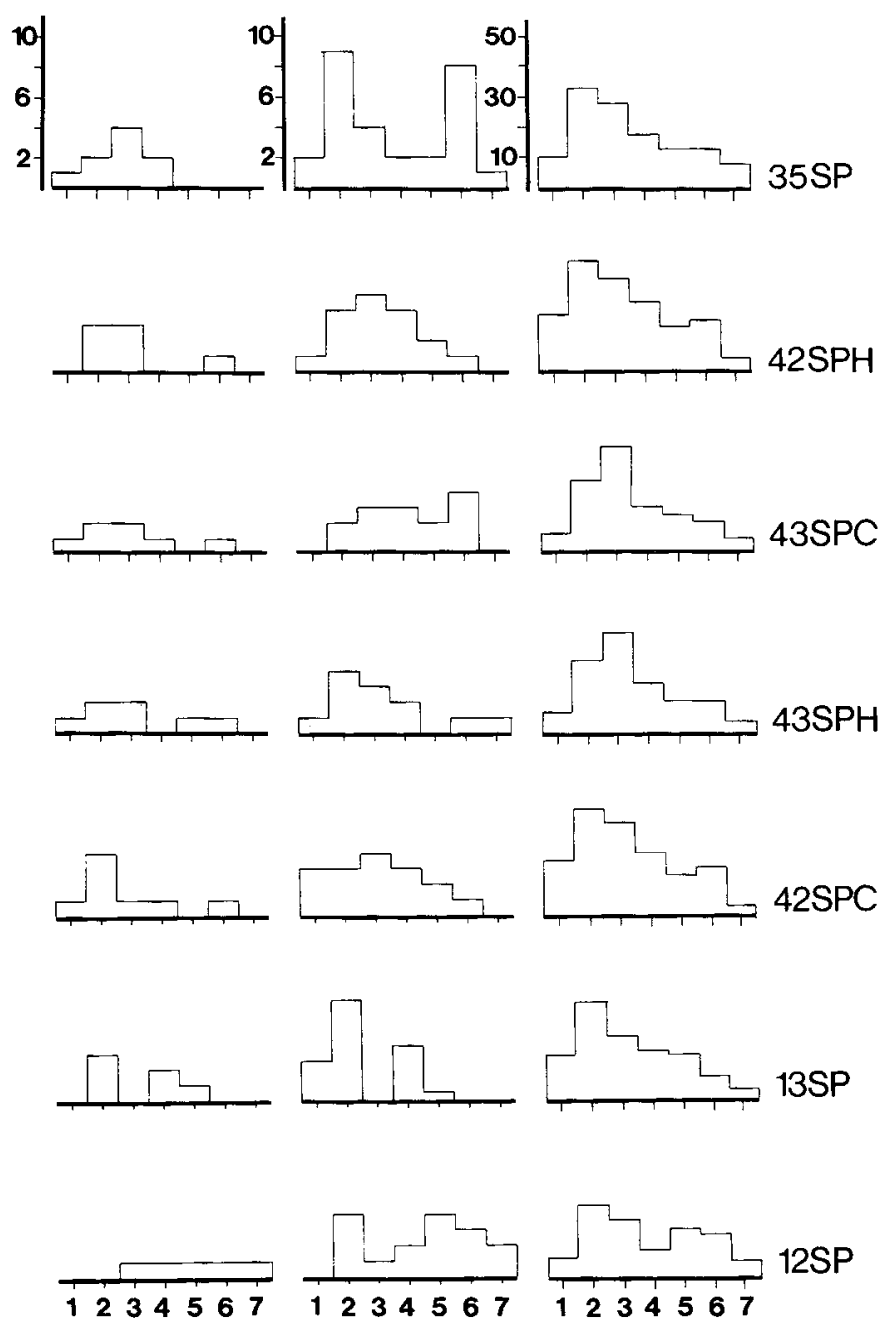

OCULAR DOMINANCE GROUP

Figure 11. Ocular dominance values of recording sites within the injected, labeled, and control regions from 7 injections. Ocular dominance was scored using the 7-point scale of Hubel and Wiesel (1962). Note that injections centered over regions that were driven primarily by the contralateral eye (i.e., ocular dominance values $I-3$ ) resulted in labeled regions exhibiting 2 different patterns of ocular dominance. In 35SP, we found that the labeled areas exhibited responses evoked by either eye. In $42 \mathrm{SPH}$, the majority of the labeled areas possessed responses driven primarily by the contralateral eye. Most of the injections resulted in labeled regions with ocular dominance values that were not significantly different from those of the controls.

others in which numerous electrode penetrations (between 80 and 120) were made to obtain a physiological map.

\section{Injections centered over regions with nonoverlapping orientation domains}

In contrast to the overlap in the patterns of labeling observed in 42SP, we found that 2 injections placed into nonoverlapping orientation domains resulted in nonoverlapping patterns of labeling. We injected 2 regions with nonoverlapping orientation values in 43SP. The retrogradely labeled patches of WGA-HRP and Con A that resulted from the 2 injections interdigitated with each other such that a quasiuniform strip of labeled cells was seen in the region between the 2 injection sites (Fig. 15).
The injection of Con A into 43SP, described in detail earlier (Figs. 8, 9), was placed into a region with a mean orientation slightly off horizontal $\left(161^{\circ} \pm 22^{\circ}\right)$. The injection of HRP was placed $1.7 \mathrm{~mm}$ anterior to the Con A injection site in a region with a mean orientation near vertical $\left(95^{\circ} \pm 24^{\circ}\right)$. The cumulative charts of labeled cells are shown in Figure 15 and illustrate that the 2 tracers were found in separate patches, quite unlike the pattern of labeling observed for 42SP (Fig. 12). Notice that in the region between the 2 injection sites the patches of label interdigitate. This is clearly shown in Figure 16, which presents a dark-field photomicrograph of the WGA-HRP injection site and labeled patches $(A)$ and a bright-field photomicrograph of the Con A injection site and labeled patches $(B)$. Arrowheads mark common blood vessels to aid in alignment. Close inspection of 43SP revealed that small, unlabeled regions between the interdigitated patches of labeling were present and may be due to an incomplete set of preferred orientations within the 2 injection sites. Nevertheless, most of the cortical surface between these 2 injections was labeled, which suggests that virtually all regions of the cortex may participate in local, intrinsic connections.

\section{Discussion}

Patterns of intrinsic connections and iso-orientation domains

From our studies of cat visual cortex, as well as of the tree shrew (Rockland and Lund, 1982; Sesma et al., 1984), ferret (Rockland, 1986), and primate (Rockland, 1985), a generalized description of cortical intrinsic circuitry emerges. Local connections are made between groups or patches of interconnected neurons that are arranged periodically across the cortical surface with a distinct center-to-center spacing, cortical range, and laminar distribution. This internal organization of periodic connections appears to be a feature unique to cortical structures, and complements the patchy and periodic pattern of the corticocortical and thalamocortical projections (LeVay and Gilbert, 1976; Goldman and Nauta, 1977).

Exact measurements show that the center-to-center spacing of the intrinsic patches in visual cortex is specific to both the species and the cortical area under study. In area V1 of the tree shrew and ferret, the spacing of the intrinsic patches is roughly $500 \mu \mathrm{m}$ (Rockland et al., 1982; Sesma et al., 1984; Rockland, 1985, 1986). In Saimiri, a New World monkey, it is about 400 $\mu \mathrm{m}$ in area V1 and $500 \mu \mathrm{m}$ in V2, while in the macaque it is $500 \mu \mathrm{m}$ in area V1 and $650 \mu \mathrm{m}$ in V2 (Rockland and Lund, 1983; Rockland, 1985). It is noteworthy that in all visual areas that have been examined there is a striking similarity in the periodicity of the intrinsic patches and in that of the isoorientation domains, as inferred from the results of 2deoxyglucose studies. In the tree shrew the iso-orientation domains have a repeat period of $500 \mu \mathrm{m}$ (Humphrey et al., 1980); in V1 of the macaque the periodicity is $570 \mu \mathrm{m}$ (Hubel et al., 1977), and in V1 of Saimiri the repeat period is $500 \mu \mathrm{m}$ (Humphrey and Hendrickson, 1983). This similarity, in itself, is suggestive of a relationship between the iso-orientation domains and intracortical patches.

In area 18 of the cat we have found that the spacing between the centers of labeled patches is roughly $1 \mathrm{~mm}$ (Fig. $5 B$ ), similar to the $1.25 \mathrm{~mm}$ obtained for the periodicity of the iso-orientation domains in area 18 (Cynader et al., 1987). We have shown that the intrinsic connections link together cells with preferred orientation values that are different and, on average, orthogonal 


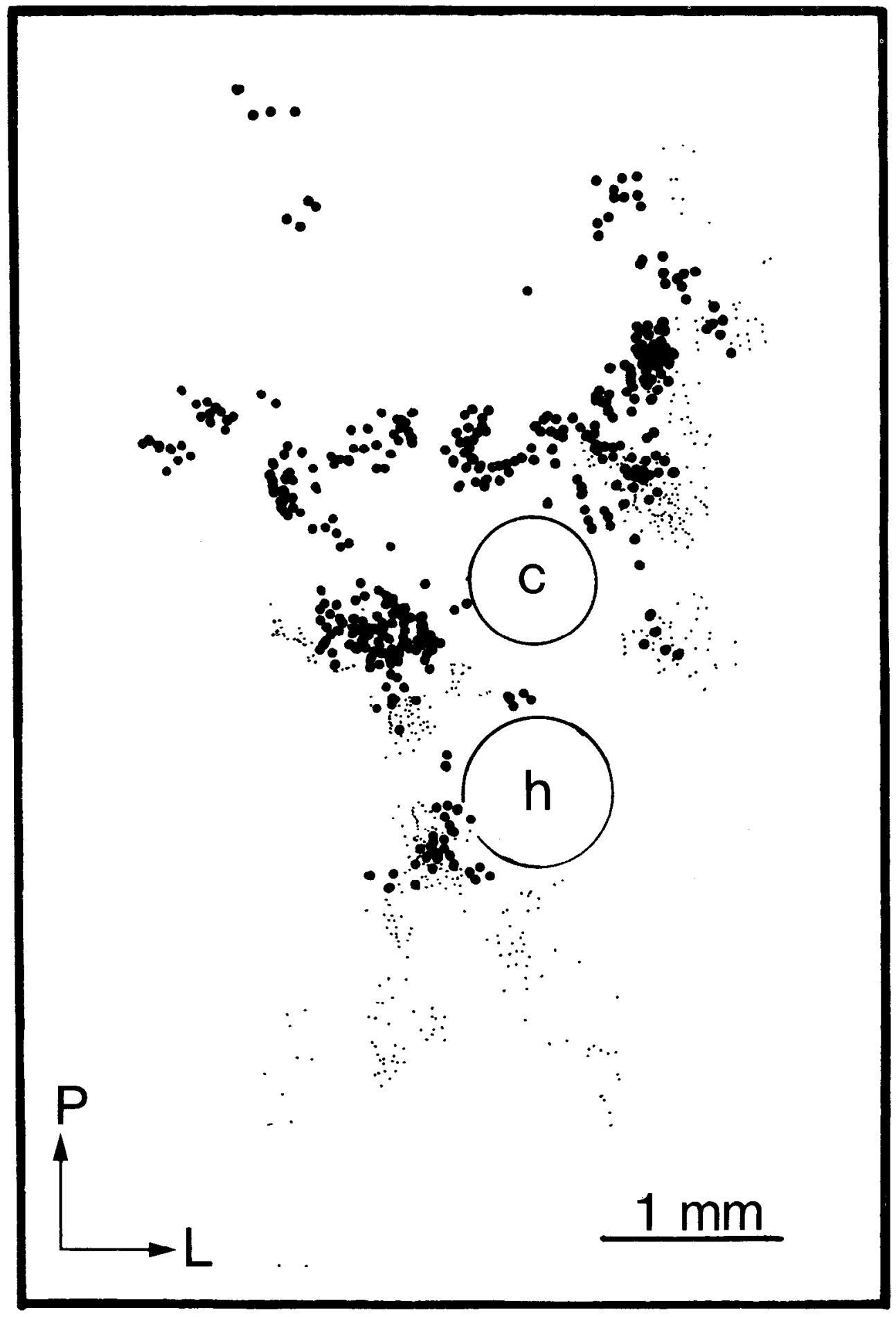

Figure 12. Cumulative charts of labeling from 2 injections in 42SP. The injections $(h=$ WGA-HRP, $c=$ Con A) were placed in overlapping orientation domains (see Figs. 13, 14) and there was extensive overlap between some of the labeled patches. Notice that the extent of the labeling is greater in the anterior-posterior than the mediallateral direction. Alternate sections were processed for either Con A or WGAHRP. Positions of neurons labeled with WGA-HRP or Con A were identified under bright-field at $400 \times$ and marked with small and large dots, respectively.

to each other. The specificity of this finding is evident, since regions with preferred orientations, like those of the injection site, are "within range," yet not labeled. The majority of the connections observed are between sets of cells with different preferred orientation values. However, we have not been able to rule out the possibility that some of these connections link together areas with similar preferred orientation values. This can be seen most readily in the cumulative histogram of Figure $10 \mathrm{~A}$. Here, the normalized difference angles between the mean orientation of the injection site and the individual orientation values of the injected, labeled, and control regions from 7 injections are shown. Note that there is some overlap between the orientation range of the injected areas and the normalized difference angle between the injected and labeled regions. At this time, we cannot be sure whether this subset of our data represents a connection between cells with similar preferred orientations or merely reflects a smearing in the results, due perhaps to connections between broadly tuned neurons or to an effective injection site that has a larger spread than the one observed histologically. Nevertheless, it is clear from our data 


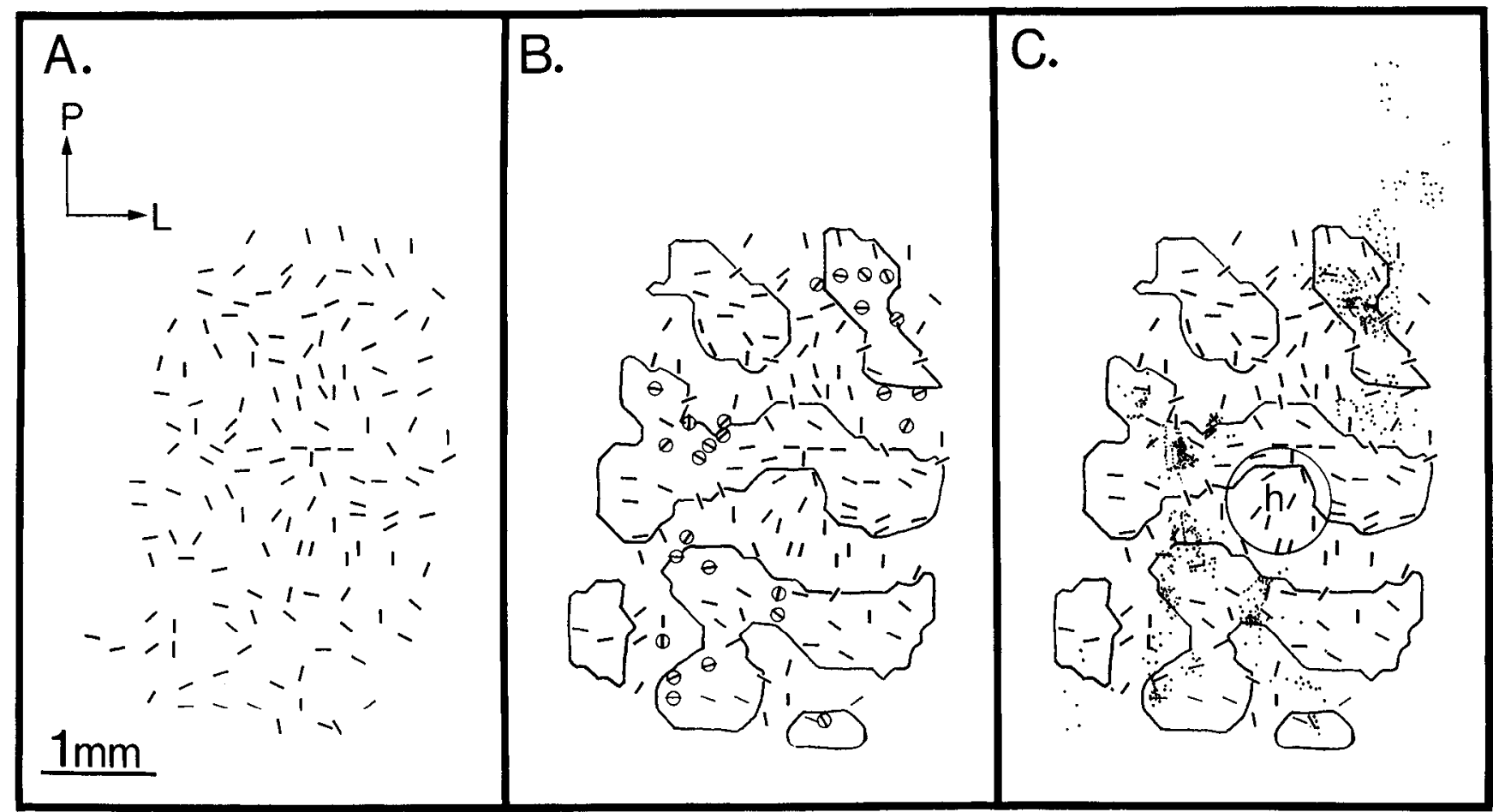

Figure 13. Preferred orientation values of 42SP are marked over the recording sites as in Figure 8 . The contours outline areas with orientation values between $60^{\circ}$ and $-45^{\circ}$. Injections of WGA-HRP and Con A were made into nearby points with overlapping orientation ranges, as shown here and in Figure 14. The range of orientations in the HRP injection site was broad $\left(69^{\circ} \pm 33^{\circ}\right)$. The labeled HRP cells were found in regions primarily within the iso-orientation domain orthogonal to the injected area. The mean orientation of the labeled regions was $22^{\circ}\left( \pm 36.8^{\circ}\right.$ ). The preferred orientations of the surrounding unlabeled regions (i.e., control area) are also illustrated.

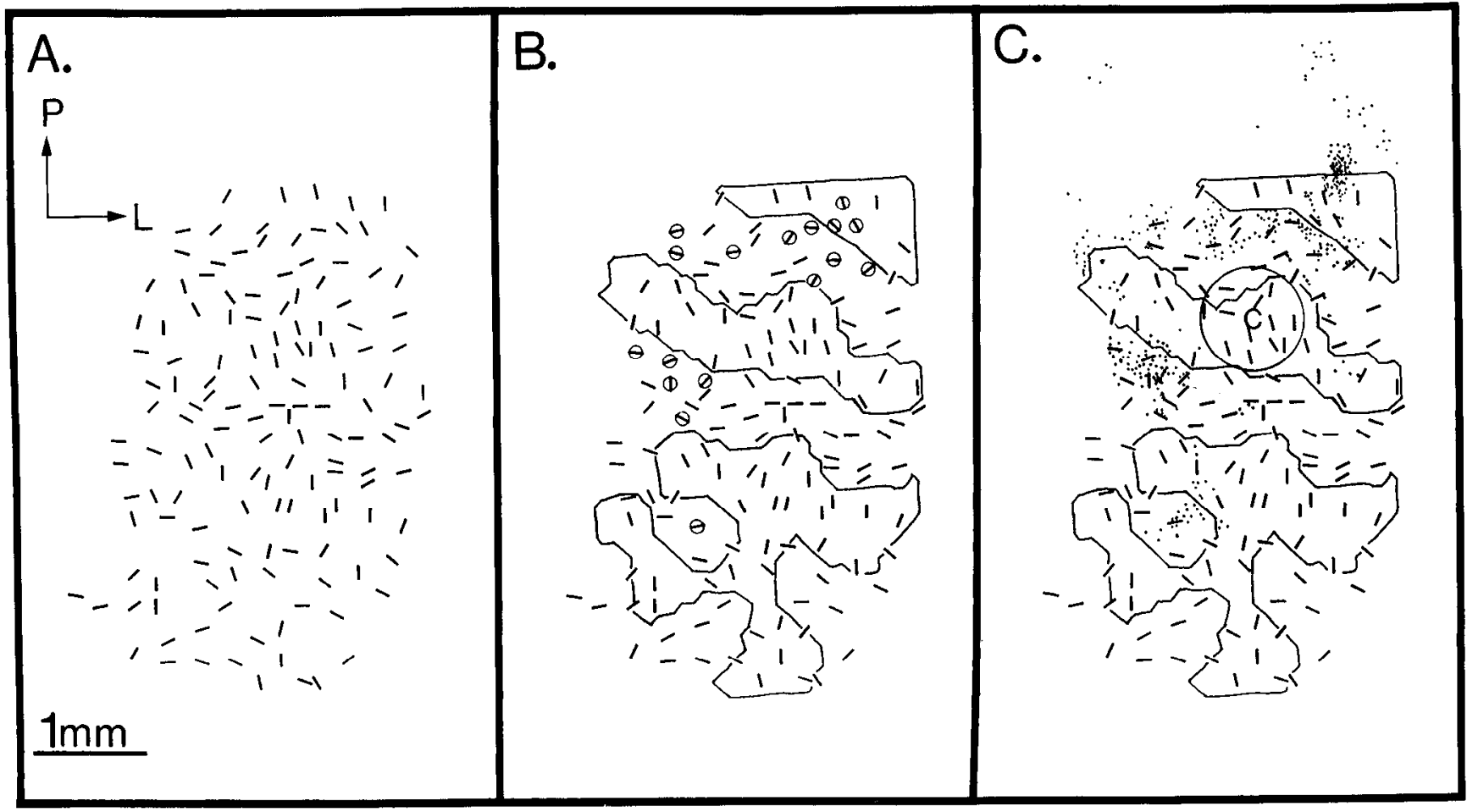

Figure 14. Preferred orientation values of the Con A injection site and labeled regions in 42SP. The contours outline orientation values between $42^{\circ}$ and $120^{\circ}$. Con $A$ was injected into an area with a mean orientation of $93^{\circ}\left( \pm 33^{\circ}\right)$. Labeling was found in regions within the iso-orientation domain orthogonal to that injected (i.e., in the areas outside the contours). The mean preferred orientation of the labeled regions was $7^{\circ}\left( \pm 35.6^{\circ}\right)$. Cumulative charts of both tracers are shown in Figure 12. 


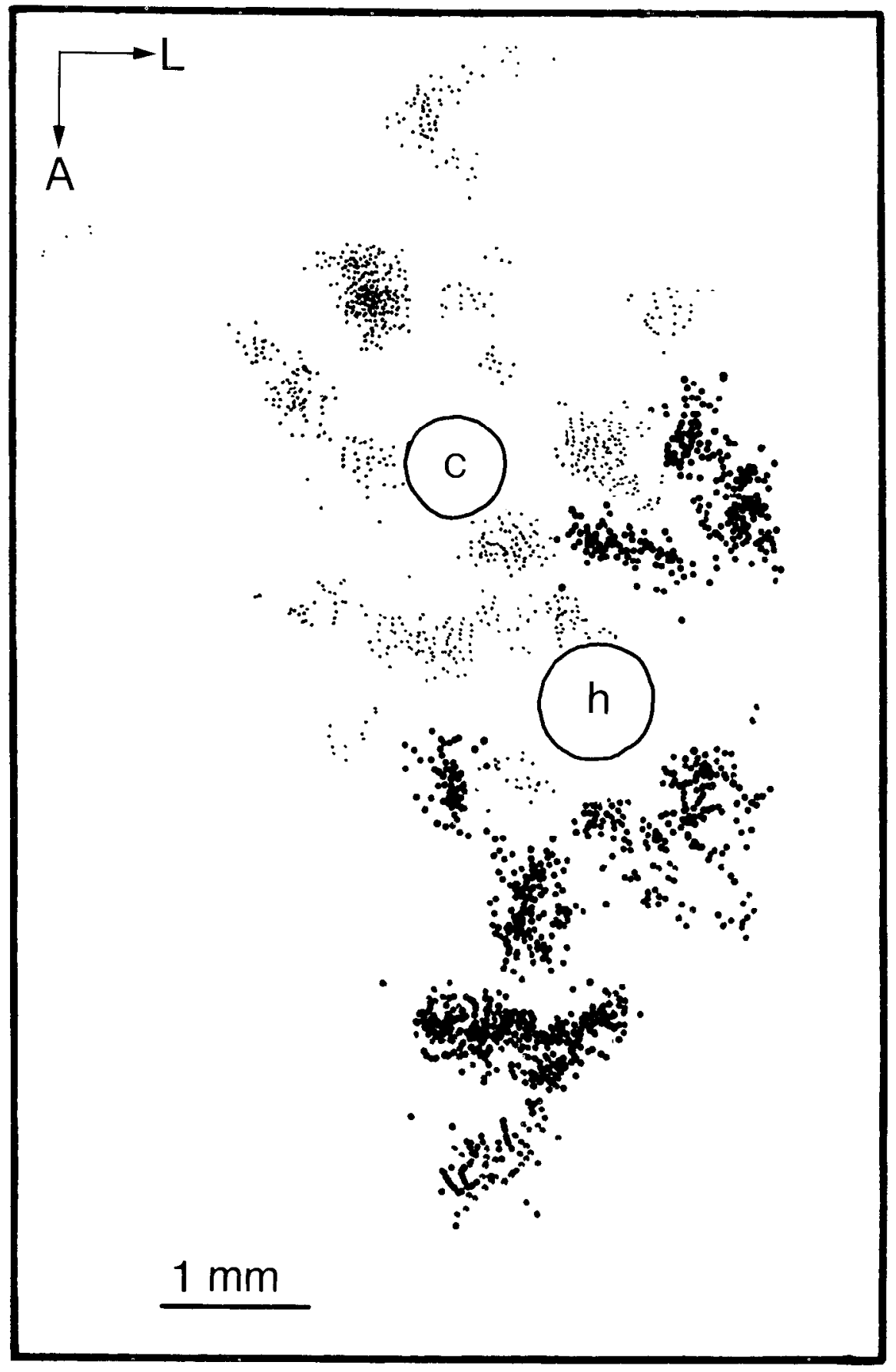

Figure 15. Pattern of labeling from an injection of WGA-HRP (large dots) and Con A (small dots) into 43SP. The sections were processed and labeled cells identified as described in Figure 12. Injections were placed in nonoverlapping orientation domains (see Fig. 8 for the orientation values of the Con A injection). The mean preferred orientation of the HRP injection site was near vertical $\left(95^{\circ} \pm 24^{\circ}\right)$. The mean value of the Con A injection was near horizontal $\left(161^{\circ} \pm 22^{\circ}\right)$. Note that the patterns of labeling from the 2 tracers have distinctly nonoverlapping distributions, quite unlike the pattern observed in Figure 12. $\mathrm{As}$ in Figure 12, the extent of labeling is greater in the anteriorposterior direction.

that most of the intrinsic connections in area 18 of the cat are between regions of cortex that have different, and most often orthogonal, preferred orientations.

Our observations are in conflict with the conclusions of Ts'o et al. (1986), who used the method of cross-correlation analysis to detect connections between pairs of simultaneously recorded cells some millimeters apart. This method shows correlations in activity (evoked by visual stimulation) only between cells with similar preferred orientation, and thus Ts'o et al. conclude that most long-range intracortical connections are between cells with similar preferred orientation. These conclusions are problematic, however. In itself, the cross-correlation method cannot distinguish a direct monosynaptic connection between a pair of cells from the presence of common inputs (e.g., from the lateral geniculate nucleus or from other cells in the same or other cortical areas). Such common inputs are almost certain to exist in the case of cell pairs with overlapping receptive fields (as was often the case in Ts'o et al.'s experiments), and their presence cannot be excluded even with cell pairs whose receptive fields do not overlap. In an experiment attempting to distinguish between common input and direct connections, Ts'o et al. (1985) reported that cell pairs whose activity was correlated when stimulated visually were no longer correlated when 1 cell of the pair was directly excited by application of a glutamate analog. This suggests that common input, rather than a direct excitatory connection between the cells, was responsible for their correlation.

Another objection to the use of the correlation method as a way of establishing the nature of intracortical connections is iis 


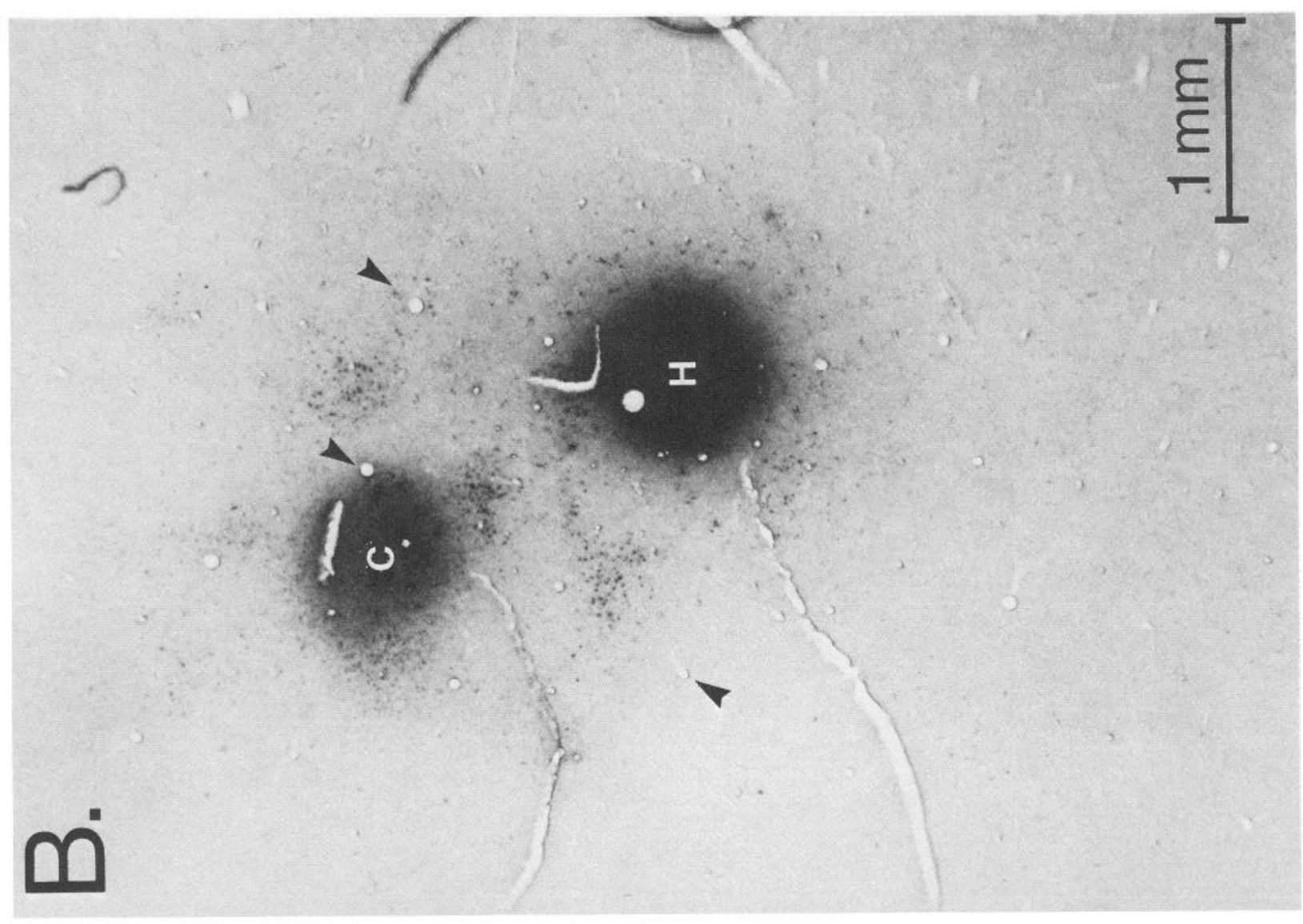

울 웅

ธี 今

हैं

递응

s.

$F$ 政.

幽要卷

ธ。

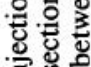

奄要

훙영.

ปี

할.

들

$\infty \leq$

ष.

Б울

0 ํ.

च

흘

․․․

㐘

手市

उं.

㱐

문

i.

仓氶

3 安

엉

I

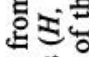

of

㲾

s.

of.

定宣寻

究 동

党

E.

․ㅡㅁ

응

密

당

政

类《

등

땡ㅇㅇㅇ

웅

至

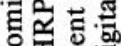

둥

훙웅

는

获

. 娄要

눙ํㄴ

行氙

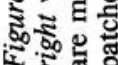




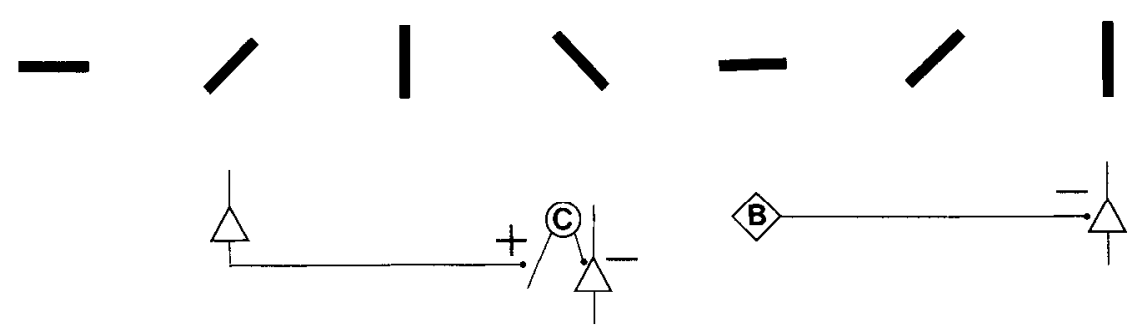

Figure 17. Results from this study suggest 2 circuits for intrinsic, patchy connections. The schematic diagram illustrates across-column connections between regions with opposite preferred orientations: Pyramidal cells (triangles) may project to inhibitory interneurons (such as a chandelier cell, $C$ ), which then inhibit neurons within their own vertical column. Alternatively, other interneurons, such as the basket cell $(B)$, may project across orientation columns and inhibit neurons directly.

relative insensitivity to the presence of inhibitory inputs (Aertsen and Gerstein, 1985). If we assume that the connections we have observed between cells with different orientation preferences are inhibitory, it is not surprising that the correlation method would fail to detect them. Common geniculate inputs to orthogonally oriented cell pairs would also be hard to detect because few, if any, visual stimuli would be capable to exciting both cells simultaneously, and reciprocal inhibition would ensure that when 1 cell fires, the other is silent.

We suggest, therefore, that the correlation method, as used by Ts'o et al. (1986) mainly reveals (1) the presence of common inputs to cell pairs that are capable of responding simultaneously to a visual stimulus, i.e., those that have similar preferred orientation, and that (2) the method fails to detect the action of the majority of long-range intracortical connections because most are inhibitory and between cells that (as a result) rarely fire simultaneously when visually stimulated.

Many other experimental results have suggested that inhibitory intracortical conncctions are present between cells with dissimilar orientation preferences. For example, intracellular recording methods revealed that presentations of nonoptimal orientations elicited IPSPs in neurons recorded in area 17 of the cat (Creutzfeldt et al., 1974). Other neurophysiological studies, in which the discharge level of extracellularly recorded single neurons is elevated by iontophoresis of excitatory amino acids, indicate that orientations outside the preferred range dramatically reduce the maintained discharge level of these cells (Ramoa et al., 1986). Moreover, neuropharmacological studies provide evidence that orientation selectivity of cortical neurons is enhanced by inhibition mediated by the neurotransmitter GABA. These studies have shown that the application of bicuculline, a GABA antagonist, dramatically affects the breadth of orientation tuning of both complex and simple cells in cat visual cortex (Sillito, 1975, 1979; Tsumoto et al., 1979). These workers also observed that in other cells the application of GABA antagonists merely reduced orientation selectivity, which suggests that intracortical inhibition enhances orientation selectivity initially provided by an excitatory convergence from lateral geniculate cells (Hubel and Wiesel, 1962). Hence, it appears that intracortical inhibition by cells tuned to orientations other than that of the recorded cell is important for generating or sharpening orientation selectivity.

While there is general agreement that inhibition plays a crucial role in the formation of orientation selectivity, there is more uncertainty regarding the range of orientations that might give rise to inhibition. Morrone et al. (1982) found that the firing rates of cells in area 17 of the cat were inhibited equally by all orientations outside the cell's tuning band. Intuitively, it seems that a broad range would be better than a narrow, strictly orthogonal range, since it can more effectively depress the flanks of a tuning curve. Given the broad orientation range of the labeled regions and considering the breadth of tuning of each of the labeled cells in these regions, we cannot distinguish the difference between an orthogonal rule and one such as that proposed by Morrone et al. (1982), in which orientation selectivity is enhanced by inhibitory connections between cells with a broad orientation range outside the range of preferred orientations of the recorded cell. Figure $10 \mathrm{~A}$ indicates that the range of difference angles between injected and labeled regions is broad enough to accommodate the results of Morrone et al. (1982).

Since not all areas with preferred orientations orthogonal to those of the injection site and "within range" were connected, criteria other than preferred orientation may be used to select out and interconnect specific areas of cortex. The nature of these additional criteria remains unknown.

Correlations between the intrinsic pattern of labeling and other receptive-field properties

\section{Magnification factor in area 18}

The anisotropic distribution of patches surrounding an injection site exists in area V2 of both the squirrel monkey and the macaque (Rockland, 1985) in addition to, as we have shown here, area 18 (V2) of the cat. These findings are consistent with earlier accounts of the anisotropic distribution of axonal fields of neurons that were based on Golgi-impregnated and silver-stained material (Colonnier, 1964) and anterograde degeneration (Creutzfeldt et al., 1977; Colonnier and Sas, 1978). The axis of maximum spread of the local connections is anterior-posterior in the cat, dorsal-ventral in the macaque, and medial-lateral in the squirrel monkey. In all these species this direction is parallel to the V1/V2 border.

Our data on the magnification factor in area 18 of the cat indicate that the representation of the visual field is anisotropic: The magnification factor for the vertical component of receptive-field position is about twice that for the horizontal component (Cynader et al., 1987). Thus, the intrinsic connections in area 18 effectively compensate for the anisotropy in magnification factor in such a way that the overall receptive field of a population of interconnected neurons is roughly circular. If this is true, the elongation in the range of intrinsic connections in the anterior-posterior direction will not be present in other cortical areas, such as area 17 , in which the magnification factor is roughly isotropic.

Preliminary studies on the development of the pattern of intrinsic connections in the cat indicated that the patches of 
labeled cells surrounding a HRP injection are formed by the third week postnatally (Price, 1986). From this study it seems that the anisotropy in the range of the connections is present from the outset. Although not mentioned by Price, it is evident from the reconstructions (fig. 3, Price, 1985) that, even during postnatal week 3 , the pattern of labeling extends further along the anterior-posterior than the medial-lateral axis, as we have found in the adult.

We have observed that along the anterior-posterior axis there is a tendency for the labeled neurons to occur at greater distances from the injection site in the posterior than the anterior direction. Since the magnification factor $\left(\mathrm{mm}^{2} / \mathrm{deg}\right.$ visual field) decreases from central to peripheral regions, the local connections may extend further in the posterior (i.e., towards the horizontal meridian) than the anterior direction in order for the set of interconnected cells to occupy a roughly symmetric topographic representation of the visual field around the injection site. It could also be argued that since the injections were placed closer to the anterior border of area 18 (i.e., in the cortical area representing the lower visual fields), the majority of the internal connections would necessarily have to be made posterior to the injection site in order to stay within the same cortical area.

Mitchison and Crick (1982) postulated that the pattern of patchy intrinsic connections was dependent on both the preferred orientation of the injected cells and the topography of visual space within the area studied. They suggested that the axis of elongation of the labeling pattern would be different for injections placed in horizontally oriented versus vertically oriented regions of cortex. Our results, however, indicate that the axis of elongation is consistently anterior-posterior regardless of the preferred orientation of the injection site (Figs. 6 $A, 7$ ).

\section{Ocular dominance}

At the outset of our studies, we speculated that the property of eye preference might play a significant role in predicting which regions of area 18 would be interconnected. We were, however, unable to detect any significant correlation between the ocular dominance values of the recording sites within the injected and the labeled regions. There are many explanations for this result. The first that occurs is related to the ocular dominance scale. The 7-point scale originally used by Hubel and Wiesel (1962) scores neurons that show no obvious difference in the excitatory effects exerted by the 2 eyes in the center of the scale (group 4). The ends of the scale (groups 1 and 7) represent "monocular" neurons (i.e., neurons driven exclusively by the contralateral or ipsilateral eye, respectively). The 7-point ocular dominance scale measures the relative contribution of cach cyc independently to the overall activity of a cortical neuron. As such, this scale is somewhat misleading, since it excludes the effects of binocular interactions such as inhibition or facilitation. It has been shown that neurons in the 1 or 7 categories (so-called "monocular" cells) exhibit an inhibitory influence from the "silent" eye and neurons in the intermediate groups (ocular dominance values $2,3,5,6)$ may exhibit varying degrees of inhibition and facilitation from the weaker eye (Kato et al., 1981). Thus, it seems that there is no direct correspondence between the ocular dominance scale and the degree of inhibition or facilitation exerted by the weaker (or silent) eye. A different measure, one in which activity arising from binocular interactions is also taken into account, may be necessary in order to uncover correlations between the cortical position of the intrinsic connections and the surface distribution of the physiological property of eye pref- erence. Alternatively, binocularity in visual cortex may be generated through nonpatchy connections acting both within and between adjacent ocular dominance columns.

\section{Vertical organization of the intrinsic patches}

\section{Laminar distribution}

The laminar distribution of cells within the intrinsic patches varies among species as well as cortical areas. In area 18 of the cat, the intrinsic patches are composed of labeled cell bodies located in all 6 layers and in columnar register. In coronal sections, the lateral extent of the cells within layer IV is less than in the supragranular and especially the infragranular layers. The spread of cells in the lower layers is consistent with findings that the axonal arbors and collaterals of layer V and VI cells span greater distances than those of cells in the superficial layers. In addition, physiological recordings indicate that the size of the receptive fields of neurons in layer IV is considerably smaller than those of neurons in the infragranular layers.

\section{Models of local circuitry}

Both pyramidal and nonpyramidal cells are present in the intrinsic patches in area 18 of the cat (Figs. 3, 4). Our studies reealed that only $2-9 \%$ of the cells within a patch were labeled after HRP injections. The implication of this finding is that relatively few cells within a cortical column are directly involved with the processing of information between columns. In one proposed circuit, pyramidal cells might interconnect orthogonal orientation columns by making excitatory contacts with known inhibitory neurons, such as the multipolar and chandelier cells (C in Fig. 17), which act within the same vertical column. Thus, multipolar and chandelier cells would have an orientation preference orthogonal to the preferred orientation of the other cells in the same column. There is physiological evidence for the existence of a small proportion of cells with preferred orientations orthogonal to the other cells within the same vertical column (Lee et al., 1977).

In a second proposed circuit, basket cells (B in Fig. 17) might interconnect adjacent columns in an inhibitory manner. It has been shown that basket cells have long, myelinated, laterally directed axons and axonal arbors that are distributed in a patchy fashion far from the soma itself (i.e., across columns) (Martin et al., 1983). These cells appear to be inhibitory interneurons, as they make symmetric synapses upon the soma and proximal dendrites of pyramidal cells (Jones, 1975; Peters and Regidor, 1981; DeFelipe and Fairen, 1982). Preliminary immunocytochemical experiments (Matsubara et al., 1987) have revealed numcrous long scgments of myclinatcd axons, coursing latcrally in the supragranular layers of area 18 , that stain intensely with an antibody directed against GABA. These labeled axons are likely to be from basket cells and indicate that across-column inhibition is far more prevalent than was once believed.

\section{Horizontal organization of the intrinsic connections}

\section{Continuous versus fixed loci}

In 3 animals we injected both succinyl Con A and WGA-HRP into physiologically identified regions of area 18 . These experiments resulted in regions of labeling that overlapped when the injections were placed into areas with similar orientation ranges, and interdigitated when the 2 tracers were injected into orthogonal orientation zones. These experiments bear directly on the question of fixed versus continuous intrinsic connectivity in visual cortex. Rockland and Lund $(1982,1983)$ recently pro- 
posed that the intrinsic connections are a fixed lattice that links together only certain cortical locations. We observed 2 patterns of labeling from our double-tracer experiments. The first consisted of HRP- or Con A-labeled neurons positioned in slightly overlapping regions, while the second pattern consisted of HRPor Con A-labeled neurons in separate but adjoining patches. Both patterns support the hypothesis of a continuous, horizontal system of connections in visual cortex. We failed to obscrve a fixed latticelike or reticular pattern such as the ones seen by Rockland and Lund (1982, 1983) and Sesma et al. (1984) in the visual cortex of the primate and tree shrew. This difference in the appearance of the intrinsic patches could reflect a speciesspecific difference in the internal organization of the visual cortex in cat and primate. However, our methods were not identical to those used in the earlier studies and may well account for the differences. The injections in the earlier studies were larger than ours and included lysolecithin, a demyelinating agent. On the basis of comparisons of numbers of cells in the lateral geniculate nucleus labeled from microinjections in the cortex, we have observed that the portion of the injection site that appears most "effective" is the central region damaged by the micropipette. Hence, we believe that the uptake and transport by cells in the halo of the injection site is significantly less than that by cells in the damaged region of the injection site. Our results indicate that the majority of the intrinsic patches occur close to the injection site (i.e., within $1 \mathrm{~mm}$ of the center). Thus, the halo of large cortical injections may obscure many of the intrinsic patches. This, combined with the use of lysolecithin, a demyelinating agent that may increase labeling of axons of passage, may have enabled these earlier workers to tap into a system of connections quite separate from the intrinsic connections that we have studied. The latticelike patterns may identify a partial subset of the intrinsic connections (i.e., those few patches occurring outside the halo of the injection site) and a second, periodic system of afferents or efferents arising from fibers of passage.

Our results from area 18 of the cat favor the continuous horizontal connection hypothesis and suggest that all regions of the cortical surface participate in the intrinsic connectivity of visual cortex. The finding that less than $10 \%$ of the neurons in a column are labeled after an injection suggests that most of the cells within a given cortical column are either local interneurons, confined to their own vertical column, or extrinsic neurons that project to other cortical areas. Recent studies using injections of retrograde tracers into other visual cortical areas indicate that the projection neurons are distributed periodically (Bullier et al., 1984; Matsubara ct al., 1984). Our data suggcst that cortical projection neurons and the long-range intrinsic neurons may not form segregated, separate columns but, rather, represent 2 overlapping and periodic systems.

\section{References}

Aertsen, A., and G. L. Gerstein (1985) Evaluation of neuronal connectivity: Sensitivity of cross-correlation. Brain Res. 340: 341-354.

Albus, K., and R. Beckman (1980) Second and third visual areas of the cat: Interindividual variability in retinotopic arrangement and cortical location. J. Physiol. (Lond.) 299: 247-276.

Batschelet, E. (1981) Circular Statistics in Biology, Academic, London.

Beaulieu, C., and M. Colonnier (1984) A comparison of the number of neurons in seven cortical areas of the cat. Soc. Neurosci. Abstr. 138: 8 .

Bullier, J., H. Kennedy, and W. Salinger (1984) Branching and laminar origin of projections between visual cortical areas in the cat. J. Comp. Neurol. 228: 329-341.
Colonnier, M. (1964) The tangential organization of the visual cortex. J. Anat. 98: 327-344.

Colonnier, M., and E. Sas (1978) An anterograde degeneration study of the tangential spread of axons in cortical areas 17 and 18 of the squirrel monkey (Saimiri sciureus). J. Comp. Neurol. 179: 245-262.

Creutzfeldt, O. D., U. Kuhnt, and L. A. Benevento (1974) An intracellular analysis of visual cortical neurones to moving stimuli: Responses in a co-operative neuronal network. Exp. Brain Res. 21:251274.

Creutzfeldt, O. D., L. J. Garey, R. Kuroda, and J.-R. Wolff (1977) The distribution of degenerating axons after small lesions in the intact and isolated visual cortex of the cat. Exp. Brain Res. 27: 419-440.

Cynader, M. S., N. V. Swindale, and J. A. Matsubara (1987) Functional topography in cat area 18. J. Neurosci. 7:1401-1413.

DeFelipe, J., and A. Fairen (1982) A type of basket cell in superficial layers of the cat visual cortex. A Golgi-electron microscope study. Brain Res. 244: 9-16.

Goldman, P., and W. H. Nauta (1977) Columnar distribution of corticocortical fibers in frontal association, limbic and motor cortex of developing rhesus monkey. Brain Res. 122: 393-413.

Goldman-Rakic, P. S., and M. L. Schwartz (1982) Interdigitation of contralateral and ipsilateral columnar projections to frontal association cortex in primates. Science 216: 755-757.

Harvey, A. R. (1980) The afferent connexions and laminar distribution of cells in area 18 of the cat. J. Physiol. (Lond.) 302: 483-505.

Horton, J. C., and D. H. Hubel (1981) Regular patchy distribution of cytochrome oxidase staining in primary visual cortex of macaque monkey. Nature 292: 762-764.

Hubel, D. H., and T. N. Wiesel (1962) Receptive fields, binocular interaction and functional architecture in the cat's visual cortex. J. Physiol. (Lond.) 160: 106-154.

Hubel, D. H., and T. N. Wiesel (1972) Laminar and columnar distribution of geniculocortical fibers in the macaque monkey. J. Comp. Neurol. 146: 421-450.

Hubel, D. H., T. N. Wiesel, and M. P. Stryker (1977) Orientation columns in macaque monkey visual cortex demonstrated by the 2-deoxyglucose autoradiographic technique. Nature 269: 328-330.

Humphrey, A. L., and A. F. Hendrickson (1983) Background and stimulus-induced patterns of high metabolic activity in the visual cortex (area 17) of the squirrel and macaque monkey. J. Neurosci. 3: 345-358.

Humphrey, A. L., L. C. Skeen, and T. T. Norton (1980) Topographic organization of the orientation column system in the striate cortex of the tree shrew (Tupaia glis). II. Deoxyglucose mapping. J. Comp. Neurol. 192: 549-566.

Humphrey, A. L., M. Sur, D. J. Uhlrich, and S. M. Sherman (1985) Termination patterns of individual $\mathrm{X}$ - and $\mathrm{Y}$-cell axons in the visual cortex of the cat: Projections to area 18, to the $17 / 18$ border region, and to both areas 17 and 18. J. Comp. Neurol. 233: 190-212.

Imig, T., and J. Brugge (1978) Sources and terminations of callosal axons related to binaural and frequency maps in primary auditory cortex of the cat. J. Comp. Neurol. 182: 637-660.

Jones, E. G. (1975) Varieties and distribution of non-pyramidal cells in the somatic sensory cortex of the squirrel monkey. J. Comp. Neurol. 160: 205-268.

Kato, H., P. O. Bishop, and G. A. Orban (1981) Binocular interaction of monocularly discharged lateral geniculate and striate neurons in the cat. J. Neurophysiol. 46: 932-951.

Lee, B. B., K. Albus, P. Heggelund, M. J. Holme, and O. D. Creutzfeldt (1977) The depth distribution of optimal stimulus orientations for neurones in cat area 17. Exp. Brain Res. 27: 301-314.

LeVay, S., and C. D. Gilbert (1976) Laminar patterns of geniculocortical projection in the cat. Brain Res. 113: 1-19.

LeVay, S., D. H. Hubel, and T. N. Wiesel (1975) The pattern of ocular dominance columns in macaque visual cortex revealed by a reduced silver stain. J. Comp. Neurol. 159: 559-576.

Livingstone, M. S., and D. H. Hubel (1982) Thalamic inputs to cytochrome oxidase rich regions in monkey visual cortex. Proc. Natl. Acad. Sci. USA 79: 6098-6101.

Livingstone, M. S., and D. H. Hubel (1984) Specificity of intrinsic connections in primate primary visual cortex. J. Neurosci. 4: 28302835.

Martin, K. A. C., P. Somogyi, and D. Whitteridge (1983) Physiological and morphological properties of identified basket cells in the cat's visual cortex. Exp. Brain Res. 50: 193-200. 
Matsubara, J. A., and D. P. Phillips (1986) Intrinsic patchy connections in primary auditory cortex of the cat. Soc. Neurosci. Abstr. 1296.

Matsubara, J. A., M. Cynader, and N. V. Swindale (1984) Physiological correlates of the anatomical connections within and between areas 17 and 18 of cat visual cortex. Soc. Neurosci. Abstr. 237: 6 .

Matsubara, J., M. Cynader, N. V. Swindale, and M. P. Stryker (1985) Intrinsic projections within visual cortex: Evidence for orientationspecific local connections. Proc. Natl. Acad. Sci. USA 82: 935-939.

Matsubara, J. A., D. M. Nance, and M. S. Cynader (1987) Laminar distribution of GABA-immunoreactive neurons and processes in area 18 of the cat. Br. Res. Bull. 18: 1-5.

Mesulam, M. M. (1978) Tetramethyl benzidine for horseradich peroxidase neurohistochemistry. J. Histochem. Cytochem. 26: 106-117.

Mitchison, G., and F. Crick (1982) Long axons within the striate cortex: Their distribution, orientation, and patterns of connection. Proc. Natl. Acad. Sci. USA 79: 3661-3665.

Moore, B. R. (1980) A modification of the Rayleigh test for vector data. Biometrika 67: 175-180.

Morrone, M. C., D. C. Burr, and L. Maffei (1982) Functional implications of cross-orientation inhibition of cortical visual cells. I. Neurophysiological evidence. Proc. R. Soc. Lond. [Biol.] 216: 335-354.

Mountcastle, V. (1957) Modality and topographic properties of single neurons of cat's somatic sensory cortex. J. Neurophysiol. 20: 408434

Nance, D. M. (1984) Succinyl concanavalin A as a neuroanatomical tracer. Soc. Neurosci. Abstr. 126: 421.

Pearse, A. G. (1972) Histochemistry. Theoretical and Applied, vol. 2, Churchill Livingstone, London.

Peters, A., and J. J. Regidor (1981) A reassessment of the forms of non-pyramidal neurons in area 17 of the cat visual cortex. J. Comp. Neurol. 203: 685-716.

Price, D. J. (1984) Patterns of cytochrome oxidase activity in areas 17,18 and 19 of the visual cortex of cats and kittens. Exp. Brain Res. 159: $1-9$.

Price, D. J. (1986) The post-natal development of clustered intrinsic connections in area 18 of the visual cortex in kittens. Dev. Brain Res. 24: $31-38$.

Ramoa, A. S., M. Shadlen, B. C. Skottun, and R. D. Freeman (1986) A comparison of inhibition of orientation and spatial frequency selectivity of cat visual cortex. Nature 321: 237-239.

Rockland, K. S. (1985) A reticular pattern of intrinsic connections in primate area V2 (area 18). J. Comp. Neurol. 235: 467-478.

Rockland, K. (1986) Anatomical organization of primary visual cortex (area 17) in the ferret. J. Comp. Neurol. 241: 225-236.
Rockland, K. S., and J. S. Lund (1982) Widespread periodic intrinsic connections in the tree shrew visual cortex. Science 215: 1532-1534.

Rockland, K. S., and J. S. Lund (1983) Intrinsic laminar lattice connections in primate visual cortex. J. Comp. Neurol. 216: 303-318.

Rockland, K. S., J. S. Lund, and A. L. Humphrey (1982) Anatomical banding of intrinsic connections in striate cortex of tree shrews (Tupaia glis). J. Comp. Neurol. 209: 41-58.

Rye, D. B., C. B. Sapel, and B. Wainer (1984) Stabilization of the tetramethylbenzidine (TMB) reaction product: Application for retrograde and anterograde tracing and combination with immunohistochemistry. J. Histochem. Cytochem. 32: 1145-1153.

Sesma, M. A., V. A. Casagrande, and J. H. Kaas (1984) Cortical connections of area 17 in tree shrews. J. Comp. Neurol. 230: 337351.

Sillito, A. M. (1975) The contribution of inhibitory mechanisms to the receptive field properties of neurons in the striate cortex of the cat. J. Physiol. (Lond.) 250: 305-329.

Sillito, A. M. (1979) Inhibitory mechanisms influencing complex cell orientation selectivity and their modification at high resting discharge levels. J. Physiol. (Lond.) 289: 33-53.

Swindale, N. V. (1982) A model for the formation of orientation columns. Proc. R. Soc. Lond. [Biol.] 215: 211-230.

Swindale, N. V. (1985) Iso-orientation domains and their relationship with cytochrome oxidase patches. In Models of the Visual Cortex, D. Rose and V. Dobson, eds., pp. 452-461, Wiley, New York.

Swindale, N. V., J. A. Matsubara, and M. S. Cynader (1987) Surface organization of orientation and direction selectivity in cat area $18 . \mathrm{J}$ Neurosci. 7: 1414-1427.

Symonds, L. L., and A. C. Rosenquist (1984) Laminar origins of visual corticocortical connections in the cat. J. Comp. Neurol. 229:39-47.

Ts'o, D. Y., C. D. Gilbert, and T. N. Wiesel (1985) Relationships between horizontal connections and functional architecture in cat striate cortex as revealed by cross correlation analysis. Soc. Neurosci. Abstr. 10: 17.

Ts'o, D. Y., C. D. Gilbert, and T. N. Wiesel (1986) Relationships between horizontal interactions and functional architecture in cat striate cortex as revealed by cross correlation analysis. J. Neurosci. 6: $1160-1170$

Tsumoto, T., W. Eckart, and O. D. Creutzfeldt (1979) Modification of orientation sensitivity of cat visual cortex neurons by removal of GABA-mediated inhibition. Exp. Brain Res. 34: 351-363.

Tusa, R. L., A. C. Rosenquist, and L. A. Palmer (1979) Retinotopic organization of areas 18 and 19 in the cat. J. Comp. Neurol. 185 . $657-678$. 